\title{
A nonperturbative calculation of nonlinear spectroscopic signals in liquid solution
}

\author{
Being J. Ka and Eitan Geva ${ }^{\text {a) }}$ \\ Department of Chemistry, University of Michigan, 930 North University, Ann Arbor, \\ Michigan 48109-1055
}

(Received 4 August 2006; accepted 11 September 2006; published online 1 December 2006)

\begin{abstract}
Nonlinear spectroscopic signals in liquid solution were calculated without treating the field-matter interaction in a perturbative manner. The calculation is based on the assumption that the intermolecular degrees of freedom can be treated classically, while the time evolution of the electronic state is treated quantum mechanically. The calculated overall electronic polarization is then resolved into its directional components via the method of Seidner et al. [J. Chem. Phys. 103, 3998 (1995)]. It is shown that the time dependence of the directional components is independent of laser intensity in the impulsive pulse regime, which allows for flexibility in choosing the procedure for calculating optical response functions. The utility and robustness of the nonperturbative procedure is demonstrated in the case of a two-state chromophore solvated in a monoatomic liquid, by calculating nonlinear time-domain signals in the strong-field, weak-field, impulsive, and nonimpulsive regimes. (C) 2006 American Institute of Physics.
\end{abstract}

[DOI: $10.1063 / 1.2359440]$

\section{INTRODUCTION}

The development of ultrafast lasers has given rise to many nonlinear time-domain techniques which are capable of probing molecular dynamics on the femtosecond time scale. ${ }^{1-8}$ All of these nonlinear optical spectroscopic techniques measure the laser-induced electronic and/or vibrational polarization of the material system, which is affected by the underlying molecular dynamics as well as by the laser pulse properties, such as intensity, carrier frequency, shape, and chirp. However, the interpretation of these experiments is usually based on a perturbative treatment of the fieldmatter interaction. ${ }^{1,9-18}$ In this case, one employs the perturbative expansion of the optical response in orders of the external driving field. ${ }^{1}$ The even-order terms in this expansion generally vanish in systems with inversion symmetry, such as liquid solutions. The first-order term is generally given in terms of a time integral over the linear optical response function (ORF), which is proportional to the quantummechanical dipole autocorrelation function. The third-order term is generally given by a sum of three-dimensional time integrals over several nonlinear third-order ORFs, which are given in terms of quantum-mechanical three-time dipole correlation functions and so on. Within the perturbative regime, one expects the linear-response optical signal to be significantly larger than the nonlinear-response signal. However, signals associated with different ORFs scatter in different directions, which makes it possible to resolve them experimentally and provides a convenient theoretical framework for classifying them according to the direction along which they are detected.

The exact calculation of ORFs is only possible in the case of relatively simple models that involve harmonic po-

${ }^{\text {a)} E l e c t r o n i c ~ m a i l: ~ e i t a n @ u m i c h . e d u ~}$ tential energy surfaces. Thus, the calculation of ORFs in anharmonic many-body systems, such as liquid solutions, has to rely on approximate methods that treat the nuclear degrees of freedom (DOFs) in a classical-like manner. ${ }^{1,10-12,14,15,17,19-23}$ When using the perturbative approach it is also commonly assumed that the pulses can be treated as nonoverlapping and impulsive, i.e., short compared to the time scale of the nuclear motion. This assumption amounts to treating the pulses as delta functions and therefore makes it possible to avoid the rather computationally costly calculation of multidimensional time integrals over the ORFs mentioned above.

A nonperturbative treatment of the field-matter interaction in the context of ultrafast nonlinear spectroscopy was considered in several previous studies. ${ }^{24-39}$ With the exception of Refs. 36-39, these studies were focused on the twopulse pump-probe technique as a tool for studying intramolecular dynamics. Furthermore, with the exception of Refs. 36 and 37 , previous studies were based on fully quantummechanical calculations carried out on low-dimensional model systems. Attempts to account for solvent effects were either based on phenomenological Bloch equations, ${ }^{38,39}$ the semigroup approach, ${ }^{26,35}$ or the assumption that the solvent can be described as consisting of independent harmonic modes whose effect can be described in terms of either Redfield theory ${ }^{30}$ or the surrogate Hamiltonian approach. ${ }^{34,35}$ A nonperturbative study of optical response in liquid solution that was based on explicit molecular dynamics simulations was reported in Refs. 36 and 37. In this case, the third- and fifth-order nonlinear Raman responses in neat liquid $\mathrm{CS}_{2}$ were calculated via nonequilibrium molecular dynamics (MD) simulations. However, in this case the Raman signal can be treated within a classical framework. The actual calculations reported in Refs. 36 and 37 also employed weak 
and impulsive pulses, and other types of nonlinear signals were not considered.

In the present paper we consider a general nonperturbative approach for calculating nonlinear signals in liquid solutions and similar anharmonic many-body systems. To this end, we avoid treating the field-matter interaction as a small perturbation and adopt a mixed quantum-classical approach that deals with the intermolecular nuclear DOFs classically, while the intramolecular electronic and/or vibrational DOFs are treated quantum mechanically.

The remainder of this paper is organized as follows. The basic model Hamiltonian and other general considerations are discussed in Sec. II. The nonperturbative procedure for calculating nonlinear spectroscopic signals is outlined in Sec. III. A demonstrative application to liquid solutions is presented in Sec. IV. Concluding remarks are presented in Sec. V.

\section{PRELIMINARY CONSIDERATIONS}

Consider a system with the following overall quantummechanical Hamiltonian:

$$
\hat{H}(t)=\hat{H}_{0}+\hat{W}(t)
$$

where $\hat{H}_{0}$ is the molecular (field-free) Hamiltonian and $\hat{W}(t)$ is the laser-matter interaction Hamiltonian. For the sake of simplicity, we present the formalism in terms of electronic spectroscopy and assume that the system can be described in terms of only two electronic states, such that

$$
\begin{aligned}
\hat{H}_{0} & =\hat{H}_{g} \otimes|g\rangle\left\langle g\left|+\left(\hbar \omega_{e g}+\hat{H}_{e}\right) \otimes\right| e\right\rangle\langle e| \\
& \equiv \hat{H}_{g}+\hbar \omega_{e g}|e\rangle\langle e|+\hat{U}| e\rangle\langle e| .
\end{aligned}
$$

Here, $|g\rangle$ and $|e\rangle$ correspond to the ground and excited electronic states, respectively, $\omega_{e g}$ corresponds to the frequency of the bare electronic transition, $\hat{H}_{g}$ and $\hat{H}_{e}$ represent the (typically multidimensional and pronouncedly anharmonic) nuclear Hamiltonians of the ground and excited electronic states, respectively, and $\hat{U}=\hat{H}_{e}-\hat{H}_{g}$. It should be emphasized that this formulation will apply equally well in the case of vibrational spectroscopy and that it can be extended to cases involving multiple electronic and/or vibrational transitions.

Assuming that the laser electric field can be described as a classical time-dependent driving force and that the electric dipole approximation is valid, the field-matter interaction Hamiltonian is given by

$$
\hat{W}(t)=-\boldsymbol{\mu}_{0}[|e\rangle\langle g|+| g\rangle\langle e|] \cdot \sum_{j} \boldsymbol{\epsilon}_{j}(t) \cos \left(\omega_{j} t-\mathbf{k}_{j} \cdot \mathbf{r}\right)
$$

Here, we have assumed that the incident laser field can be expressed as a sum of several phase-locked transformlimited pulses, such that $\boldsymbol{\epsilon}_{j}(t), \omega_{j}$, and $\mathbf{k}_{j}$ are the envelope, leading frequency, and wave vector of the $j$ th pulse, respectively, and $\boldsymbol{\mu}_{0}[|e\rangle\langle g|+| g\rangle\langle e|]$ is the molecular dipole moment operator. For the sake of simplicity, we will restrict ourselves to cases where the leading frequency is the same for all pulses, $\omega_{j}=\omega$, and where the Condon and rotating wave ap- proximations are valid. Under these conditions, one may put $\hat{W}(t)$ in the following form: $:^{1,17}$

$$
\hat{W}(t)=-\frac{\hbar}{2} \sum_{j}\left(\chi_{j}(t) e^{i \omega t}|g\rangle\left\langle e\left|+\chi_{j}^{*}(t) e^{-i \omega t}\right| e\right\rangle\langle g|\right),
$$

where $\hbar \chi_{j}(t)=\boldsymbol{\mu}_{0} \cdot \boldsymbol{\epsilon}_{j}(t) e^{-i \mathbf{k}_{j} \cdot \mathbf{r}}$.

We denote the density operator of the overall system at time $t$ by $\hat{\rho}(t)$. The time evolution of $\hat{\rho}(t)$ is dictated by the following equation of motion:

$$
\frac{d}{d t} \hat{\rho}(t)=-\frac{i}{\hbar}[\hat{H}(t), \hat{\rho}(t)] .
$$

If $\hbar \omega_{e g} / k_{B} T \gg 1$, where $T$ is the absolute temperature, then one may assume that the initial state of the overall system is given by

$$
\hat{\rho}(t=0)=\hat{\rho}_{g}^{\mathrm{eq}}|g\rangle\langle g|,
$$

where $\hat{\rho}_{g}^{\text {eq }}=e^{-\beta \hat{H}_{g}} / \operatorname{Tr}_{N}\left(e^{-\beta \hat{H}_{g}}\right)\left(\beta=1 / k_{B} T\right.$ and $\operatorname{Tr}_{N}$ is the trace with respect to the nuclear DOF).

Equation (5) is given in terms of the Schrödinger picture. An alternative representation in terms of the rotating frame (with respect to the electronic DOFs) and interaction picture (with respect to the nuclear DOFs) will prove more convenient for our purposes. The density operator of the overall system in this new representation is given by

$$
\begin{aligned}
\tilde{\rho}(t)= & \exp \left[i\left(\hat{H}_{g}+\hbar \omega|e\rangle\langle e|\right) t / \hbar\right] \hat{\rho}(t) \\
& \times \exp \left[-i\left(\hat{H}_{g}+\hbar \omega|e\rangle\langle e|\right) t / \hbar\right] .
\end{aligned}
$$

It can be easily shown that the equation of motion for $\widetilde{\rho}(t)$ is given by ${ }^{17}$

$$
\frac{d}{d t} \tilde{\rho}(t)=-\frac{i}{\hbar}[\tilde{H}(t), \tilde{\rho}(t)],
$$

where

$$
\tilde{H}(t)=(\hbar \Delta+\hat{U}(t))|e\rangle\langle e|-\frac{\hbar}{2} \sum_{j}\left(\chi_{j}(t)|g\rangle\left\langle e\left|+\chi_{j}^{*}(t)\right| e\right\rangle\langle g|\right) .
$$

Here, $\hat{U}(t)=e^{i \hat{H}_{g} t / \hbar} \hat{U} e^{-i \hat{H}_{g} t / \hbar}$ and $\Delta=\omega_{e g}-\omega$ is the detuning. For the sake of simplicity, we will restrict ourselves from this point on to resonance conditions, namely, to $\Delta=0$.

\section{CALCULATION OF NONLINEAR SPECTROSCOPIC SIGNALS}

Within the framework of the dipole and Condon approximations, the electronic polarization $P(t)$ is proportional to the real part of the expectation value of the electronic coherence operator $|e\rangle\langle g|$ :

$$
\begin{aligned}
P(t) & \propto \operatorname{Re}(\operatorname{Tr}[|e\rangle\langle g| \hat{\rho}(t)]) \\
& =\operatorname{Re}\left(\left\langle g\left|\operatorname{Tr}_{N}[\hat{\rho}(t)]\right| e\right\rangle\right) \\
& =\operatorname{Re}(\langle g|\hat{\sigma}(t)| e\rangle) \\
& =\operatorname{Re}\left(\sigma_{g e}(t)\right) \\
& =\operatorname{Re}\left(e^{i \omega t} \widetilde{\sigma}_{g e}(t)\right) .
\end{aligned}
$$


Here, $\operatorname{Tr}$ is the trace over nuclear and electronic DOFs, $\hat{\sigma}(t)=\operatorname{Tr}_{N}[\hat{\rho}(t)]$ is the reduced density operator that describes the state of the electronic DOF in the Schrödinger picture, and $\widetilde{\sigma}(t)=e^{i \omega|e\rangle\langle e| t} \hat{\sigma}(t) e^{-i \omega|e\rangle\langle e| t}$ is its counterpart in the rotating frame representation. Within the thin sample approximation and homodyne-detection scheme, ${ }^{1,29}$ the measurable signal at time $t$, denoted by $S(t)$, is proportional to $|P(t)|^{2}$ averaged over the period of the driving field, which implies that

$$
S(t) \propto\left|\widetilde{\sigma}_{g e}(t)\right|^{2} .
$$

The coupled equations of motion for the four elements of the density matrix $\widetilde{\sigma}(t)$ are given by

$$
\begin{aligned}
& \frac{d \widetilde{\sigma}_{g e}}{d t}=\left(\frac{d \widetilde{\sigma}_{e g}}{d t}\right)^{*}=i U(t) \widetilde{\sigma}_{g e}(t) / \hbar+\sum_{j} \frac{i \chi_{j}(t)}{2}\left(\widetilde{\sigma}_{e e}-\widetilde{\sigma}_{g g}\right), \\
& \frac{d \widetilde{\sigma}_{e e}}{d t}=-\frac{d \widetilde{\sigma}_{g g}}{d t}=\sum_{j}\left(\frac{i \chi_{j}^{*}(t)}{2} \widetilde{\sigma}_{g e}(t)-\frac{i \chi_{j}(t)}{2} \widetilde{\sigma}_{e g}(t)\right)
\end{aligned}
$$

It should be noted that we have assumed that $\hat{U}(t)$ can be replaced by its classical counterpart, $U(t)$. The latter can be computed by monitoring the energy gap between the ground and excited electronic states during a classical equilibrium MD simulation on the ground state potential. The signal can then be calculated by averaging $\widetilde{\sigma}_{g e}(t)$, as obtained by numerically solving Eqs. (12) and (13), over a sufficiently large number of trajectories traced by $U(t)$. The assumption that $U(t)$ can be treated classically is motivated by our desire to focus on the signature of solvation dynamics on nonlinear optical signals. We can therefore assume that the fluctuations of $U(t)$ reflect translational and rotational dynamics of the solvent molecules (rather than intramolecular vibrational dynamics of the chromophore), which can usually be described in classical terms.

It should be noted that the mixed quantum-classical scheme underlying Eqs. (12) and (13) is dictated by the specific partitioning of the Hamiltonian in Eq. (2), which in turn dictates the choice of interaction picture in Eq. (7). This scheme is convenient in practice, since $U(t)$ can be calculated via equilibrium MD simulations on the ground state potential surface. However, the partitioning of the Hamiltonian in Eq. (2) is not unique, and other partition schemes are known to lead to different mixed quantum classical schemes where the time evolution of $U(t)$ is dictated by a potential energy surface that corresponds to an average of the ground and excited electronic surfaces. ${ }^{10-17}$ A more detailed discussion of this point is provided in Appendix A.

\section{A. The impulsive regime}

Many previous studies of nonlinear optical spectroscopy have been based on the assumption that the pulses are impulsive. In this case, one assumes that the pulses are very short, such that $U$ can be assumed to be fixed on the time scale of the $j$ th pulse width $\tau_{j}$. The fact that the pulses are very short also implies that one can ignore their overlap and therefore treat the effect of each pulse separately from that of the other pulses. It is also convenient to assume that the pulses have a square envelope, such that $\chi_{j}(t)=\chi_{j}$ when $t_{j} \leqslant t \leqslant t_{j}+\tau_{j}$ and zero otherwise. Under these conditions, it is straightforward to show that the time evolution during an impulsive pulse is given by

$$
\widetilde{\sigma}\left(t_{j}+\tau_{j}\right)=\mathcal{W}_{j} \widetilde{\sigma}\left(t_{j}\right) \mathcal{W}_{j}^{-1}
$$

where

$$
\begin{aligned}
& \mathcal{W}_{j}=\left\{\cos \left(\frac{\theta_{j}}{2}\right) \hat{1}-i \sin \left(\frac{\theta_{j}}{2}\right)\left[\sin \left(\eta_{j}\right)(|e\rangle\langle e|-| g\rangle\langle g|)\right.\right. \\
& \left.\left.+\cos \left(\eta_{j}\right)\left(e^{i \phi_{j}}|g\rangle\left\langle e\left|+e^{-i \phi_{j}}\right| e\right\rangle\langle g|\right)\right]\right\}, \\
& \mathcal{W}_{j}^{-1}=\left\{\cos \left(\frac{\theta_{j}}{2}\right) \hat{1}+i \sin \left(\frac{\theta_{j}}{2}\right)\left[\sin \left(\eta_{j}\right)(|e\rangle\langle e|-| g\rangle\langle g|)\right.\right. \\
& \left.\left.+\cos \left(\eta_{j}\right)\left(e^{i \phi_{j}}|g\rangle\left\langle e\left|+e^{-i \phi_{j}}\right| e\right\rangle\langle g|\right)\right]\right\} \text {. }
\end{aligned}
$$

Here, $\hat{1}$ is the identity operator, $\theta_{j}=\sqrt{\left[U\left(t_{j}\right) \tau_{j} / \hbar\right]^{2}+\left[\left|\chi_{j}\right| \tau_{j}\right]^{2}}$, $\chi_{j}=\left|\chi_{j}\right| e^{i \phi_{j}}$, and $\tan \left(\eta_{k}\right)=-U\left(t_{j}\right) / \hbar\left|\chi_{j}\right|$.

A treatment based on Eq. (15) will be referred to below as corresponding to the weak impulsive regime. It should be noted that in this case, the time evolution during the pulse is explicitly dependent on the value of $U$ at the time of the pulse. Adding the assumption that the field is strong, in the sense that $\left|\chi_{j}\right| \gg U\left(t_{j}\right) / \hbar$, would imply that $\theta_{j} \approx\left|\chi_{j}\right| \tau_{j}$ and $\eta_{k} \approx 0$, which would in turn simplify $\mathcal{W}_{j}$ and $\mathcal{W}_{j}^{-1}$ as follows:

$$
\begin{aligned}
& \mathcal{W}_{j}=\cos \left(\theta_{j} / 2\right) \hat{1}+i \sin \left(\theta_{j} / 2\right)\left[e^{i \phi_{j}}|g\rangle\left\langle e\left|+e^{-i \phi_{j}}\right| e\right\rangle\langle g|\right], \\
& \mathcal{W}_{j}^{-1}=\cos \left(\theta_{j} / 2\right) \hat{1}-i \sin \left(\theta_{j} / 2\right)\left[e^{i \phi_{j}}|g\rangle\left\langle e\left|+e^{-i \phi_{j}}\right| e\right\rangle\langle g|\right],
\end{aligned}
$$

where $\chi_{j} \tau_{j}=\theta_{j} e^{i \phi_{j}}$. A treatment which is based on Eq. (16) will be referred to below as corresponding to the strong impulsive regime.

The dynamics between the $j$ th and $(j+1)$ th pulses is obtained by setting $\chi_{j}=0$ in Eqs. (12) and (13), so that

$$
\widetilde{\sigma}\left(t_{j+1}\right)=\mathcal{G}\left(t_{j+1}, t_{j}\right) \widetilde{\sigma}\left(t_{j}\right) \mathcal{G}^{-1}\left(t_{j+1}, t_{j}\right),
$$

where

$$
\begin{aligned}
& \mathcal{G}\left(t_{j+1}, t_{j}\right)=\exp \left[-i|e\rangle\langle e| \int_{t_{j}}^{t_{j+1}} d t^{\prime} U\left(t^{\prime}\right) / \hbar\right], \\
& \mathcal{G}^{-1}\left(t_{j+1}, t_{j}\right)=\exp \left[i|e\rangle\langle e| \int_{t_{j}}^{t_{j+1}} d t^{\prime} U\left(t^{\prime}\right) / \hbar\right] .
\end{aligned}
$$

It should be noted that we assumed that $t_{j}+\tau_{j} \approx t_{j}$, since impulsive pulses are presumably very short.

Let us next consider the commonly encountered case where the system is subject to three consecutive pulses in the strong impulsive regime. We label the pulses $a, b$, and $c$. It is convenient to define the time origin so that it coincides with pulse $a$, i.e., $t_{a}=0$. We also denote the time intervals between the first and second pulses and between the second and third pulses by $t_{1}$ and $t_{2}$, respectively, so that $t_{b}=t_{1}$ and $t_{c}=t_{1}+t_{2}$. The polarization a time interval $t$ after the third pulse is proportional to $\widetilde{\sigma}_{g e}\left(t_{1}, t_{2}, t\right)$, which is given by 


$$
\begin{aligned}
\widetilde{\sigma}_{g e}\left(t_{1}, t_{2}, t\right)= & \operatorname{Tr}_{N} \rho_{g}^{\mathrm{eq}}\left\{\langle g| \mathcal{G}\left(t_{1}+t_{2}+t, t_{1}+t_{2}\right)\right. \\
& \times \mathcal{W}_{c} \mathcal{G}\left(t_{1}+t_{2}, t_{1}\right) \mathcal{W}_{b} \mathcal{G}\left(t_{1}, 0\right) \mathcal{W}_{a}|g\rangle\langle g| \\
& \times \mathcal{W}_{a}^{-1} \mathcal{G}^{-1}\left(t_{1}, 0\right) \mathcal{W}_{b}^{-1} \mathcal{G}^{-1}\left(t_{1}+t_{2}, t_{1}\right) \\
& \left.\times \mathcal{W}_{c}^{-1} \mathcal{G}^{-1}\left(t_{1}+t_{2}+t, t_{1}+t_{2}\right)|e\rangle\right\},
\end{aligned}
$$

where $\operatorname{Tr}_{N} \rho_{g}^{\text {eq }\{\cdots\}}$ should be interpreted as a classical average over the equilibrium distribution of initial solvent configurations.

Calculating $\widetilde{\sigma}_{g e}\left(t_{1}, t_{2}, t\right)$ explicitly is rather tedious, but straightforward otherwise. The final result can be written as a linear combination of nine terms:

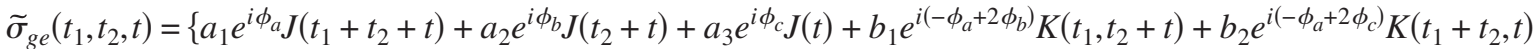

$$
\begin{aligned}
& \left.+b_{3} e^{i\left(-\phi_{b}+2 \phi_{c}\right)} K\left(t_{2}, t\right)+c_{1} e^{i\left(\phi_{a}-\phi_{b}+\phi_{c}\right)} L_{+}\left(t_{1}, t_{2}, t\right)+c_{2} e^{i\left(-\phi_{a}+\phi_{b}+\phi_{c}\right)} L_{-}\left(t_{1}, t_{2}, t\right)+d e^{i\left(\phi_{a}-2 \phi_{b}+2 \phi_{c}\right)} M\left(t_{1}, t_{2}, t\right)\right\} .
\end{aligned}
$$

The linear combination in Eq. (20) involves the following four ORFs.

- The linear ORF,

$$
J(\tau)=\left\langle\exp \left(i \int_{0}^{\tau} d \tau^{\prime} U\left(\tau^{\prime}\right) / \hbar\right)\right\rangle_{g},
$$

where $\langle\cdots\rangle_{g}=\operatorname{Tr}_{N}\left[\rho_{g}^{\text {eq } \cdots]}\right.$.

- The two-pulse echo (2PE) ORF,

$$
\begin{aligned}
K\left(\tau_{1}, \tau\right)= & \left\langle\operatorname { e x p } \left(-i \int_{0}^{\tau_{1}} d \tau^{\prime} U\left(\tau^{\prime}\right) / \hbar\right.\right. \\
& \left.\left.+i \int_{\tau_{1}}^{\tau_{1}+\tau} d \tau^{\prime} U\left(\tau^{\prime}\right) / \hbar\right)\right\rangle_{g},
\end{aligned}
$$

where $\tau_{1}$ is the time interval between the two pulses and $\tau$ is the time interval between the second pulse and detection.

- The three-pulse echo (3PE) ORFs,

$$
\begin{aligned}
L_{ \pm}\left(\tau_{1}, \tau_{2}, \tau\right)= & \left\langle\operatorname { e x p } \left( \pm i \int_{0}^{\left|\tau_{1}\right|} d \tau^{\prime} U\left(\tau^{\prime}\right) / \hbar\right.\right. \\
& \left.\left.+i \int_{\left|\tau_{1}\right|+\tau_{2}}^{\left|\tau_{1}\right|+\tau_{2}+\tau} d \tau^{\prime} U\left(\tau^{\prime}\right) / \hbar\right)\right\rangle_{g},
\end{aligned}
$$

where $\left|\tau_{1}\right|$ is the time interval between the first and second pulses, $\tau_{2}$ is the time interval between the second and third pulses, and $\tau$ is the time interval between the third pulse and detection. It should be noted that one can define a single 3PE ORF $L\left(\tau_{1}, \tau_{2}, \tau\right)$, which is equal to $L_{+}\left(\tau_{1}, \tau_{2}, \tau\right)$, if $\tau_{1} \leqslant 0$, and to $L_{-}\left(\tau_{1}, \tau_{2}, \tau\right)$, if $\tau_{1} \geqslant 0$. Thus, $L_{-}\left(\tau_{1}, \tau_{2}, \tau\right)$ and $L_{+}\left(\tau_{1}, \tau_{2}, \tau\right)$ correspond to the 3PE signal measured with $\tau_{1}>0$ (i.e., pulse $a$ precedes pulse $b$ ) or $\tau_{1}<0$ (i.e., pulse $b$ precedes pulse $a$ ), respectively.

- The fifth-order ORF,

$$
\begin{aligned}
M\left(\tau_{1}, \tau_{2}, \tau\right)= & \left\langle\operatorname { e x p } \left( i \int_{0}^{\tau_{1}} U\left(\tau^{\prime}\right) / \hbar-i \int_{\tau_{1}}^{\tau_{1}+\tau_{2}} U\left(\tau^{\prime}\right) / \hbar\right.\right. \\
& \left.\left.+i \int_{\tau_{1}+\tau_{2}}^{\tau_{1}+\tau_{2}+\tau} U\left(\tau^{\prime}\right) / \hbar\right)\right\rangle_{g}
\end{aligned}
$$

The coefficients $\left\{a_{1}, a_{2}, a_{3}, b_{1}, b_{2}, b_{3}, c_{1}, c_{2}, d\right\}$ depend on $\left\{\theta_{j}=\left|\chi_{j}\right| \tau_{j}\right\}$ and are explicitly given by

$$
\begin{aligned}
a_{1}= & i \cos ^{2}\left(\frac{\theta_{a}}{2}\right) \cos ^{2}\left(\frac{\theta_{b}}{2}\right) \cos ^{2}\left(\frac{\theta_{c}}{2}\right)\left[-\tan \left(\frac{\theta_{a}}{2}\right)\right], \\
a_{2}= & i \cos ^{2}\left(\frac{\theta_{a}}{2}\right) \cos ^{2}\left(\frac{\theta_{b}}{2}\right) \cos ^{2}\left(\frac{\theta_{c}}{2}\right)\left[1-\tan ^{2}\left(\frac{\theta_{a}}{2}\right)\right] \\
& \times\left[-\tan \left(\frac{\theta_{b}}{2}\right)\right],
\end{aligned}
$$

$$
\begin{aligned}
a_{3}= & i \cos ^{2}\left(\frac{\theta_{a}}{2}\right) \cos ^{2}\left(\frac{\theta_{b}}{2}\right) \cos ^{2}\left(\frac{\theta_{c}}{2}\right)\left[1-\tan ^{2}\left(\frac{\theta_{a}}{2}\right)\right] \\
& \times\left[1-\tan ^{2}\left(\frac{\theta_{b}}{2}\right)\right]\left[-\tan \left(\frac{\theta_{c}}{2}\right)\right], \\
b_{1}= & i \cos ^{2}\left(\frac{\theta_{a}}{2}\right) \cos ^{2}\left(\frac{\theta_{b}}{2}\right) \cos ^{2}\left(\frac{\theta_{c}}{2}\right) \tan \left(\frac{\theta_{a}}{2}\right) \tan ^{2}\left(\frac{\theta_{b}}{2}\right), \\
b_{2}= & i \cos ^{2}\left(\frac{\theta_{a}}{2}\right) \cos ^{2}\left(\frac{\theta_{b}}{2}\right) \cos ^{2}\left(\frac{\theta_{c}}{2}\right) \tan \left(\frac{\theta_{a}}{2}\right) \tan ^{2}\left(\frac{\theta_{c}}{2}\right), \\
b_{3}= & i \cos ^{2}\left(\frac{\theta_{a}}{2}\right) \cos ^{2}\left(\frac{\theta_{b}}{2}\right) \cos ^{2}\left(\frac{\theta_{c}}{2}\right) \\
& \times\left[1-\tan ^{2}\left(\frac{\theta_{a}}{2}\right)\right] \tan \left(\frac{\theta_{b}}{2}\right) \tan ^{2}\left(\frac{\theta_{c}}{2}\right),
\end{aligned}
$$




$$
\begin{aligned}
c_{1}= & c_{2}=2 i \cos ^{2}\left(\frac{\theta_{a}}{2}\right) \cos ^{2}\left(\frac{\theta_{b}}{2}\right) \cos ^{2}\left(\frac{\theta_{c}}{2}\right) \\
& \times \tan \left(\frac{\theta_{a}}{2}\right) \tan \left(\frac{\theta_{b}}{2}\right) \tan \left(\frac{\theta_{c}}{2}\right), \\
d= & i \cos ^{2}\left(\frac{\theta_{a}}{2}\right) \cos ^{2}\left(\frac{\theta_{b}}{2}\right) \cos ^{2}\left(\frac{\theta_{c}}{2}\right) \\
& \times\left[-\tan \left(\frac{\theta_{a}}{2}\right)\right] \tan ^{2}\left(\frac{\theta_{b}}{2}\right) \tan ^{2}\left(\frac{\theta_{c}}{2}\right) .
\end{aligned}
$$

It is important to note that, regardless of the pulse intensities, the overall polarization in the strong impulsive limit is given by a mixture of the same four ORFs. In fact, the ultimate goal of the experiment is to measure one or more of these ORFs. Furthermore, the individual ORFs can be ex- tracted from the overall polarization by taking advantage of the fact that they are associated with different phase factors. ${ }^{29}$ Experimentally, these phases reflect the fact that different signals correspond to different wave vectors and therefore scatter in different directions. Computationally, the phase factors $e^{i \phi_{a}}, e^{i \phi_{b}}, e^{i \phi_{c}}, e^{i\left(-\phi_{a}+2 \phi_{b}\right)}, e^{i\left(-\phi_{a}+2 \phi_{c}\right)}, e^{i\left(-\phi_{b}+2 \phi_{c}\right)}$, $e^{i\left(\phi_{a}-\phi_{b}+\phi_{c}\right)}, e^{i\left(-\phi_{a}+\phi_{b}+\phi_{c}\right)}$, and $e^{i\left(\phi_{a}-2 \phi_{b}+2 \phi_{c}\right)}$ can be though as coefficients in the linear combination of ORFs [cf. Eq. (20)]. Thus, following Seidner et al., ${ }^{29}$ one may obtain different independent linear combinations of the ORFs by changing the relative phases of the pulses. The corresponding set of linear coupled algebraic equations can then be solved for the individual ORFs.

For example, assigning the following phases for the first and second pulses $\left\{\phi_{a}, \phi_{b}=0, \pi / 2, \pi\right\}$ while holding the phase of the third pulse fixed at $\phi_{c}=\pi / 2$ yields the following set of nine coupled linear equations:

$$
\left(\begin{array}{l}
\widetilde{\sigma}_{g e}^{00(\pi / 2)}\left(t_{1}, t_{2}, t\right) \\
\widetilde{\sigma}_{g e}^{0(\pi / 2)(\pi / 2)}\left(t_{1}, t_{2}, t\right) \\
\widetilde{\sigma}_{g e}^{0 \pi(\pi / 2)}\left(t_{1}, t_{2}, t\right) \\
\widetilde{\sigma}_{g e}^{(\pi / 2) 0(\pi / 2)}\left(t_{1}, t_{2}, t\right) \\
\widetilde{\sigma}_{g e}^{(\pi / 2)(\pi / 2)(\pi / 2)}\left(t_{1}, t_{2}, t\right) \\
\widetilde{\sigma}_{g e}^{(\pi / 2) \pi(\pi / 2)}\left(t_{1}, t_{2}, t\right) \\
\widetilde{\sigma}_{g e}^{\pi(\pi)}(\pi / 2)\left(t_{1}, t_{2}, t\right) \\
\widetilde{\sigma}_{g e}^{\pi(\pi / 2)(\pi / 2)}\left(t_{1}, t_{2}, t\right) \\
\widetilde{\sigma}_{g e}^{\pi \pi(\pi / 2)}\left(t_{1}, t_{2}, t\right)
\end{array}\right)=\left(\begin{array}{ccccccccc}
1 & 1 & i & 1 & -1 & -1 & i & i & -1 \\
1 & i & i & -1 & -1 & i & 1 & -1 & 1 \\
1 & -1 & i & 1 & -1 & 1 & -i & -i & -1 \\
i & 1 & i & -i & i & -1 & -1 & 1 & -i \\
i & i & i & i & i & i & i & i & i \\
i & -1 & i & -i & i & 1 & 1 & -1 & -i \\
-1 & 1 & i & -1 & 1 & -1 & -i & -i & 1 \\
-1 & i & i & 1 & 1 & i & -1 & 1 & -1 \\
-1 & -1 & i & -1 & 1 & 1 & i & i & 1
\end{array}\right)\left(\begin{array}{c}
a_{1} J\left(t_{1}+t_{2}+t\right) \\
a_{2} J\left(t_{2}+t\right) \\
a_{3} J(t) \\
b_{1} K\left(t_{1}, t_{2}+t\right) \\
b_{2} K\left(t_{1}+t_{2}, t\right) \\
b_{3} K\left(t_{2}, t\right) \\
c_{1} L_{+}\left(t_{1}, t_{2}, t\right) \\
c_{2} L_{-}\left(t_{1}, t_{2}, t\right) \\
d M\left(t_{1}, t_{2}, t\right)
\end{array}\right) .
$$

Here, $\widetilde{\sigma}_{g e}^{\phi_{a} \phi_{b} \phi_{c}}\left(t_{1}, t_{2}, t\right)$ is the value of the overall polarization, $\widetilde{\sigma}_{g e}\left(t_{1}, t_{2}, t\right)$, obtained following three pulses with phases $\phi_{a}$, $\phi_{b}$, and $\phi_{c}$. The individual ORFs can then be obtained from these nine overall polarizations by inverting the matrix in Eq. (26) [cf. Eq. (B1) in Appendix B].

It should be noted that the above scheme treats the lasermatter interaction in a nonperturbative manner. The strength of the laser-matter interaction is hidden in $\theta_{j}=\left|\chi_{j}\right| \tau_{j}$ and therefore affects the values of the coefficients $\left\{a_{1}, a_{2}, a_{3}, b_{1}, b_{2}, b_{3}, c_{1}, c_{2}, d\right\}$. For example, in the limit of weak laser-matter interaction, one assumes that all pulses are of similar strength, i.e., $\theta_{j} \sim \theta$, and expands the above mentioned coefficients to leading order in powers of $\theta$ :

$$
\begin{aligned}
& a_{1}, a_{2}, a_{3} \rightarrow-i \theta / 2, \\
& b_{1}, b_{2}, b_{3}, c_{1} / 2, c_{2} / 2 \rightarrow i(\theta / 2)^{3} \\
& d \rightarrow-i(\theta / 2)^{5}
\end{aligned}
$$

This is obviously also the origin for the classification of the ORFs as linear, third order, or fifth order.

However, it should be noted that although weak lasermatter interaction may faithfully represent experimental reality in many cases of practical interest, the computational procedure outlined above is in no way restricted to the weakfield limit. In other words, employing unrealistically intense pulses in the calculation would still lead to the very same ORFs. For example, consider the case of intense laser pulses such that $\theta_{a}=\theta_{b}=\theta_{c}=\pi / 2$. This choice immediately eliminates three out of the nine components of the overall polarization, namely, $a_{2}=a_{3}=b_{3}=0$. However, it should be noted that no information is lost. This is because $J\left(t_{1}+t_{2}+t\right)$ contains the same information as $J\left(t_{2}+t\right)$ and $J(t)$, while $K\left(t_{2}, t\right)$ contains the same information as $K\left(t_{1}, t_{2}+t\right)$ and $K\left(t_{1}+t_{2}, t\right)$. The remaining six nonvanishing components can now be resolved by assigning the values $\{0, \pi / 2\}$ and $\{0, \pi / 2, \pi\}$ to $\phi_{a}$ and $\phi_{b}$, respectively, while holding the phase of the third pulse fixed at $\phi_{c}=\pi / 2$. This yields the following set of six coupled linear equations: 


$$
\left(\begin{array}{l}
\widetilde{\sigma}_{g e}^{00(\pi / 2)}\left(t_{1}, t_{2}, t\right) \\
\widetilde{\sigma}_{g e}^{0(\pi / 2)(\pi / 2)}\left(t_{1}, t_{2}, t\right) \\
\widetilde{\sigma}_{g e}^{0 \pi(\pi / 2)}\left(t_{1}, t_{2}, t\right) \\
\tilde{\sigma}_{g e}^{(\pi / 2) 0(\pi / 2)}\left(t_{1}, t_{2}, t\right) \\
\tilde{\sigma}_{g e}^{(\pi / 2)(\pi / 2)(\pi / 2)}\left(t_{1}, t_{2}, t\right) \\
\tilde{\sigma}_{g e}^{(\pi / 2) \pi(\pi / 2)}\left(t_{1}, t_{2}, t\right)
\end{array}\right)=\frac{i}{8}\left(\begin{array}{cccccc}
-1 & 1 & -1 & 2 i & 2 i & 1 \\
-1 & -1 & -1 & 2 & -2 & -1 \\
-1 & 1 & -1 & -2 i & -2 i & 1 \\
-i & -i & i & -2 & 2 & i \\
-i & i & i & 2 i & 2 i & -i \\
-i & -i & i & 2 & -2 & i
\end{array}\right)\left(\begin{array}{c}
J\left(t_{1}+t_{2}+t\right) \\
K\left(t_{1}, t_{2}+t\right) \\
K\left(t_{1}+t_{2}, t\right) \\
L_{+}\left(t_{1}, t_{2}, t\right) \\
L_{-}\left(t_{1}, t_{2}, t\right) \\
M\left(t_{1}, t_{2}, t\right)
\end{array}\right) .
$$

The individual ORFs can then be obtained by inverting the matrix in Eq. (28) [cf. Eq. (B2) in Appendix B].

The procedure can be further simplified if one is particularly interested in resolving specific components. For example, resolving the 3PE ORFs can be achieved by assigning the values $\{0, \pi / 2\}$ and $\{0, \pi\}$ for $\phi_{a}$ and $\phi_{b}$, respectively, while holding the phase of the third pulse fixed at $\phi_{c}=0$. The resulting four linear coupled equations are given by

$$
\begin{aligned}
& \left(\begin{array}{l}
\widetilde{\sigma}_{g e}^{000}\left(t_{1}, t_{2}, t\right) \\
\widetilde{\sigma}_{g e}^{0 \pi 0}\left(t_{1}, t_{2}, t\right) \\
\widetilde{\sigma}_{g e}^{(\pi / 2) 00}\left(t_{1}, t_{2}, t\right) \\
\widetilde{\sigma}_{g e}^{(\pi / 2) \pi 0}\left(t_{1}, t_{2}, t\right)
\end{array}\right)=\frac{i}{8}\left(\begin{array}{cccc}
-1 & 1 & 2 & 2 \\
-1 & 1 & -2 & -2 \\
-i & -i & 2 i & -2 i \\
-i & -i & -2 i & 2 i
\end{array}\right) \\
& \times\left(\begin{array}{c}
J\left(t_{1}+t_{2}+t\right)+M\left(t_{1}, t_{2}, t\right) \\
K\left(t_{1}, t_{2}+t\right)+K\left(t_{1}+t_{2}, t\right) \\
L_{+}\left(t_{1}, t_{2}, t\right) \\
L_{-}\left(t_{1}, t_{2}, t\right)
\end{array}\right) .
\end{aligned}
$$

As before, the individual ORFs can be obtained by inverting the matrix in Eq. (29) [cf. Eq. (B3) in Appendix B].

Extending the analysis described above to the weak impulsive regime requires that we substitute $\mathcal{W}_{j}$ and $\mathcal{W}_{j}^{-1}$ from Eq. (15) into Eq. (19). The resulting overall polarization will still consist of nine terms, with the exact same phase factors. Thus, the above procedure for resolving the overall polarization into its directional coefficients will still be valid. However, the analogous expressions for the coefficients $\left\{a_{1}, a_{2}, a_{3}, b_{1}, b_{2}, b_{3}, c_{1}, c_{2}, d\right\}$ can no longer be taken outside of the average $\langle\cdots\rangle_{g}$ because $\theta_{j}$ and $\eta_{j}$ are explicitly dependent on the values of $U$ at the time of the pulses. For example,

$$
\begin{aligned}
c_{2} L_{-}\left(t_{1}, t_{2}, t\right)= & c_{2}\left\langle\operatorname { e x p } \left(-i \int_{0}^{t_{1}} d t^{\prime} U\left(t^{\prime}\right) / \hbar\right.\right. \\
& \left.\left.+i \int_{t_{1}+t_{2}}^{t_{1}+t_{2}+t} d t^{\prime} U\left(t^{\prime}\right) / \hbar\right)\right\rangle_{g}
\end{aligned}
$$

in Eq. (20) will now be replaced by

$$
\begin{aligned}
& \left\langle c_{2}\left[U(0), U\left(t_{1}\right), U\left(t_{1}+t_{2}\right)\right]\right. \\
& \left.\quad \times \exp \left(-i \int_{0}^{t_{1}} d t^{\prime} U\left(t^{\prime}\right) / \hbar+i \int_{t_{1}+t_{2}}^{t_{1}+t_{2}+t} d t^{\prime} U\left(t^{\prime}\right) / \hbar\right)\right\rangle_{g},
\end{aligned}
$$

where

$$
c_{2}\left[U(0), U\left(t_{1}\right), U\left(t_{1}+t_{2}\right)\right]
$$

is the expression that replaces $c_{2}$ when going from the strong to the weak impulsive regime. Thus, at least in principle, $L_{-}\left(t_{1}, t_{2}, t\right)$ can no longer be extracted in a straightforward manner. However, in many cases it would be reasonable to assume that $\left\langle c_{2}\left[U(0), U\left(t_{1}\right), U\left(t_{1}+t_{2}\right)\right] \exp \left(-i \int_{0}^{t_{1}} d t^{\prime} U\left(t^{\prime}\right) / \hbar\right.\right.$ $\left.\left.+i \int_{t_{1}+t_{2}}^{t_{1}+t_{2}+t} d t^{\prime} U\left(t^{\prime}\right) / \hbar\right)\right\rangle_{g} \approx\left\langle c_{2}\left[U(0), U\left(t_{1}\right), U\left(t_{1}+t_{2}\right)\right]\right\rangle_{g}\langle\exp ($ $\left.\left.-i \int_{0}^{t_{1}} d t^{\prime} U\left(t^{\prime}\right) / \hbar+i \int_{t_{1}+t_{2}}^{t_{1}+t_{2}+t} d t^{\prime} U\left(t^{\prime}\right) / \hbar\right)\right\rangle_{g}$. Thus, the directional signal can still be proportional to $L_{-}\left(t_{1}, t_{2}, t\right)$, as long as the dependence of $\left\langle c_{2}\left[U(0), U\left(t_{1}\right), U\left(t_{1}+t_{2}\right)\right]\right\rangle_{g}$ on $t_{1}$ and $t_{2}$ is relatively weak.

\section{B. The nonimpulsive regime}

In the case of nonimpulsive pulses, one has to account for the time evolution of $U(t)$ during the pulse, which is particularly important in cases where $U(t)$ is comparable to or larger than $\hbar|\chi(t)|$. Another new aspect that needs to be accounted for in this case is the possibility that the pulses overlap in time.

The time evolution of a two-state system with a Hamiltonian $\tilde{H}(t)=U(t)|e\rangle\langle e|-\hbar \Sigma_{j}\left[\chi_{j}(t)|g\rangle\left\langle e\left|+\chi_{j}^{*}(t)\right| e\right\rangle\langle g|\right] / 2$ can be simulated by propagating the system in small time steps during which $U(t)$ and $\hbar \chi_{j}(t)$ can be assumed to be quasiconstant. Thus propagation by a small time step $\delta t$ from time $t_{k}$ to time $t_{k+1}$, during a period of time where one or more pulses are on, is given by

$$
\widetilde{\sigma}\left(t_{k+1}\right)=\mathcal{W}_{k} \widetilde{\sigma}\left(t_{k}\right) \mathcal{W}_{k}^{-1},
$$

where

$$
\begin{aligned}
& \mathcal{W}_{k}=\cos \left(\frac{\Theta_{k}}{2}\right) \hat{1}-i \sin \left(\frac{\Theta_{k}}{2}\right)\left[\sin \left(\Psi_{k}\right)(|e\rangle\langle e|-| g\rangle\langle g|)\right. \\
& \left.+\cos \left(\Psi_{k}\right)\left(e^{i \Phi_{k}}|g\rangle\left\langle e\left|+e^{-i \Phi_{k}}\right| e\right\rangle\langle g|\right)\right], \\
& \mathcal{W}_{k}^{-1}=\cos \left(\frac{\Theta_{k}}{2}\right) \hat{1}+i \sin \left(\frac{\Theta_{k}}{2}\right)\left[\sin \left(\Psi_{k}\right)(|e\rangle\langle e|-| g\rangle\langle g|)\right. \\
& \left.+\cos \left(\Psi_{k}\right)\left(e^{i \Phi_{k}}|g\rangle\left\langle e\left|+e^{-i \Phi_{k}}\right| e\right\rangle\langle g|\right)\right] .
\end{aligned}
$$

Here, $\Theta_{k}=\sqrt{\left[U\left(t_{k}\right) \delta t / \hbar\right]^{2}+\left[\left|\Xi_{k}\right| \delta t\right]^{2}}, \quad \Xi_{k}=\Sigma_{j} \chi_{j}\left(t_{k}\right)=\left|\Xi_{k}\right| e^{i \Phi_{k}}$, and $\tan \left(\Psi_{k}\right)=-U\left(t_{k}\right) / \hbar\left|\Xi_{k}\right|$. 

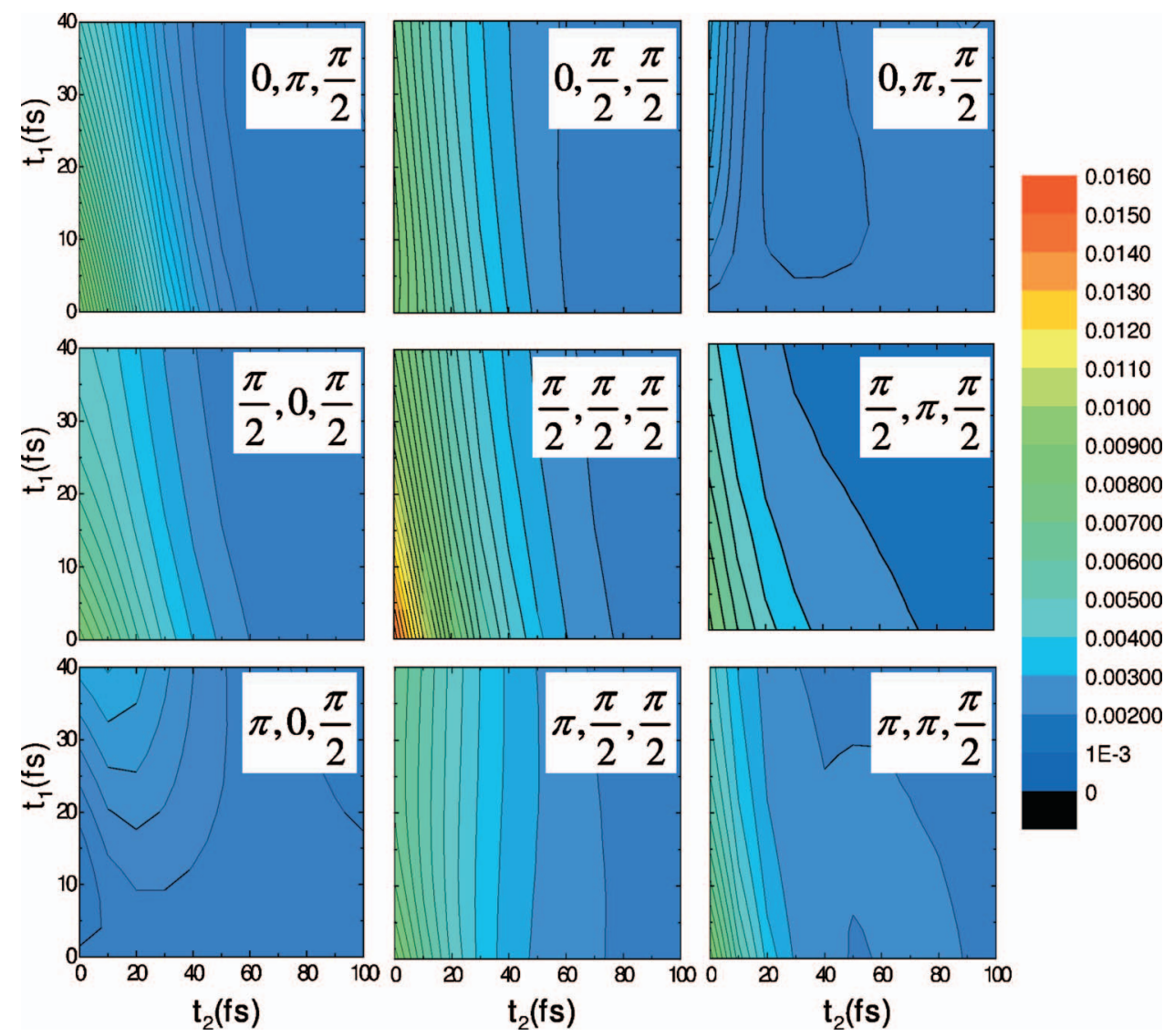

FIG. 1. (Color) The homodynedetected integrated overall polarizations, $\int_{0}^{\infty} d t\left|\widetilde{\sigma}_{g e}^{\phi_{a} \phi_{b} \phi_{c}}\left(t_{1}, t_{2}, t\right)\right|^{2}$, following a sequence of three impulsive pulses with the indicated nine different phase combinations $\left(\phi_{a}, \phi_{b}, \phi_{c}\right)$ [cf. Eq. (26)], under weak-field conditions $\left(\left|\chi_{a}\right| \tau_{a}=\left|\chi_{b}\right| \tau_{b}=\left|\chi_{c}\right| \tau_{c}=0.01 \pi / 2\right)$.

\section{APPLICATION: A TWO-STATE CHROMOPHORE IN A MONOATOMIC LIQUID}

\section{A. Model and simulation techniques}

In this section, we will test the procedures developed in Sec. III in the case of a two-state chromophore solvated in a monoatomic liquid. The potential energy surfaces of the ground and excited electronic states are assumed to be pairwise additive, such that

$$
V_{\alpha}=\sum_{k} v_{\alpha}\left(r_{k}\right)+\sum_{k<j} v_{s}\left(r_{k j}\right) .
$$

Here, $r_{k}$ is the distance between the chromophore and the $k$ th solvent atom, $r_{k j}$ is the distance between the $k$ th and $j$ th solvent atoms, and $\alpha=g, e$. It should be noted that the chromophore-solvent interactions in the excited state are assumed to be different from those in the ground state, whereas the solvent-solvent interactions in both states are assumed to be the same.

The actual pair potentials $v_{g}(r), v_{e}(r)$ and $v_{s}(r)$ were adopted from Refs. 15 and 18, where they were assumed to take the form of the Lennard-Jones (LJ) potentials:

$$
v_{j}(r)=4 \epsilon_{j}\left[\left(\frac{\sigma_{j}}{r}\right)^{12}-\left(\frac{\sigma_{j}}{r}\right)^{6}\right] .
$$

We also assume that $v_{g}(r)=v_{s}(r)$, with LJ parameters that correspond to liquid argon $\left(\epsilon / k_{B}=119.8 \mathrm{~K}, \sigma=0.3405 \mathrm{~nm}\right)$. $v_{e}(r)$ differs from $v_{g}(r)$ in the value of the LJ parameter $\sigma$, such that $\sigma_{e}=(1+\lambda) \sigma_{g}$, with $\lambda=0.06 .^{15,18}$ We also assume that the chromophore mass is the same as that of the solvent atoms and equal to the mass of an argon atom.
We note that the linear and 3PE ORFs for this model have already been reported and analyzed in Refs. 15 and 18, where they have been calculated directly based on the perturbative treatment and assuming strong impulsive pulses. However, our goal here is to demonstrate and test the nonperturbative and nonimpulsive procedure proposed above for calculating the nonlinear spectroscopic signals. Nevertheless, the results obtained via our procedure are consistent with the corresponding results from Refs. 15 and 18 in the weak-field and strong impulsive regimes (see below).

MD simulations were carried out on the ground state potential energy surface at temperature $T=128.2 \mathrm{~K}$ and density $\rho=17.98 \mathrm{~nm}^{-3}$ (the corresponding reduced LJ temperature and density are given by $T^{*}=k_{B} T / \epsilon_{s}=1.07$ and $\rho^{*}$ $=\rho \sigma_{s}^{3}=0.71$, respectively). Simulations were preformed with 108 atoms in a cubical simulation box with standard periodic boundary conditions (minimum image convention). Time propagation was carried out by the velocity Verlet method. ${ }^{40}$ The system was equilibrated over $10^{6}$ time steps, each of length $1.0 \mathrm{fs}$, by the velocity rescaling method. ${ }^{40}$ Converged results were obtained by averaging over 4000 trajectories, each of length 700-840 fs.

Once the trajectory traced by $U(t)$ is extracted from the equilibrium MD simulations outlined above, it is used in order to calculate the signals that correspond to different combinations of $\left\{t_{1}, t_{2}, t\right\}$ and $\left\{\phi_{a}, \phi_{b}, \phi_{c}\right\}$. The results reported below were obtained by varying $t_{1}$ in steps of $4 \mathrm{fs}, t_{2}$ in steps of $10 \mathrm{fs}$ and $t$ is steps of $1.0 \mathrm{fs}$ on a $10 \times 10 \times 700$ three-dimensional (3D) grid. Integration over $t$ was based on the trapezoidal rule. 

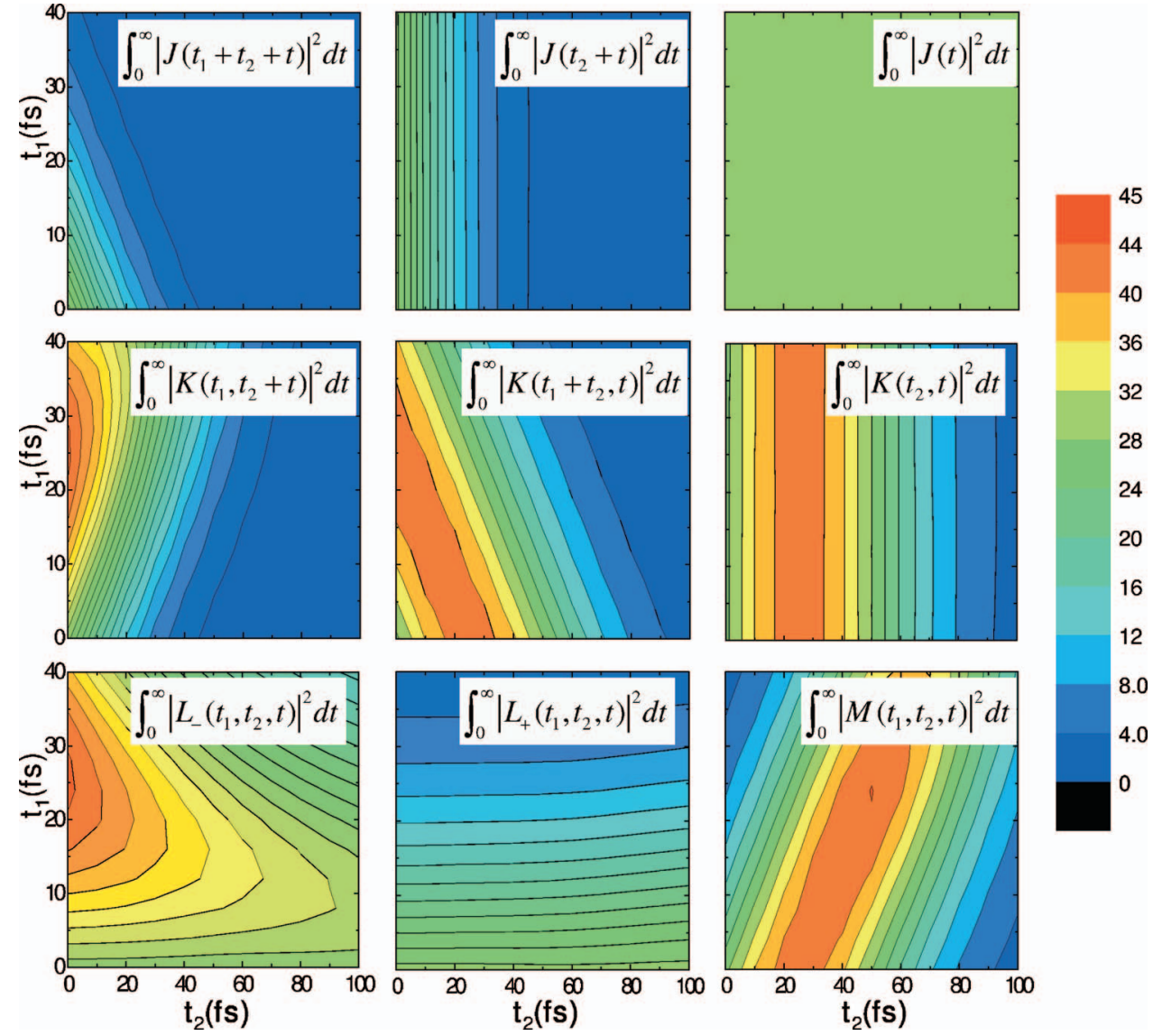

FIG. 2. (Color) The nine homodynedetected integrated ORFs as obtained via Eq. (B1), using as input the nine overall polarizations from Fig. 1.
The signal of a time-resolved heterodyne-detected threepulse experiment is a complex function of the three time intervals $t_{1}, t_{2}$, and $t$. However, in many cases, a homodynedetection scheme is employed and the signal is integrated over $t$, such that the results can be presented as a real function of $t_{1}$ and $t_{2}$ :

$$
I\left(t_{1}, t_{2}\right)=\int_{0}^{\infty} d t S\left(t_{1}, t_{2}, t\right) \propto \int_{0}^{\infty} d t\left|\widetilde{\sigma}_{g e}\left(t_{1}, t_{2}, t\right)\right|^{2} .
$$

Since this procedure is convenient for plotting the various signals, we will adopt it below when we report the results obtained from our calculations. However, it is important to note that the procedure outlined above requires the complex and $t$-resolved overall polarization as input in order to compute the various ORFs.

\section{B. The impulsive regime}

We start the discussion with a set of results obtained in the strong impulsive regime [cf. Eq. (16)], under weak-field conditions $\left(\left|\chi_{a}\right| \tau_{a}=\left|\chi_{b}\right| \tau_{b}=\left|\chi_{c}\right| \tau_{c}=0.01 \pi / 2\right)$. Figure 1 shows the integrated homodyne-detected overall polarizations obtained in this case, following a sequence of three pulses with the nine phase combinations indicated in Eq. (26). Substituting these overall polarizations into Eq. (B1) resolves the overall polarization into its directional components: $a_{1} J\left(t_{1}\right.$ $\left.+t_{2}+t\right), \quad a_{2} J\left(t_{2}+t\right), \quad a_{3} J(t), \quad b_{1} K\left(t_{1}, t_{2}+t\right), \quad b_{2} K\left(t_{1}+t_{2}, t\right)$, $b_{3} K\left(t_{2}, t\right), c_{1} L_{+}\left(t_{1}, t_{2}, t\right), c_{2} L_{-}\left(t_{1}, t_{2}, t\right)$, and $d M\left(t_{1}, t_{2}, t\right)$. The ORFs $J\left(t_{1}+t_{2}+t\right), \quad J\left(t_{2}+t\right), \quad J(t), \quad K\left(t_{1}, t_{2}+t\right), \quad K\left(t_{1}+t_{2}, t\right)$, $K\left(t_{2}, t\right), L_{+}\left(t_{1}, t_{2}, t\right), L_{-}\left(t_{1}, t_{2}, t\right)$, and $M\left(t_{1}, t_{2}, t\right)$ can be ob- tained by simply dividing by the corresponding coefficients [cf. Eq. (25)]. The modulus squares of these ORFs, integrated over $t$, which correspond to the signals measured along different directions within the homodyne timeintegrated measurement scheme, are presented in Fig. 2.

Three of the nine functions correspond to linear response to the first pulse $\left[J\left(t_{1}+t_{2}+t\right)\right]$, second pulse $\left[J\left(t_{2}+t\right)\right]$, and third pulse $[J(t)]$. Indeed, these three functions coincide with the linear ORF $J(\tau)$ when plotted as a functions of $t_{1}+t_{2}+t$, $t_{2}+t$, and $t$, respectively (not shown). It should also be noted that the rapid decay of $J(\tau)$ for the system considered here is dominated by inhomogeneous broadening (i.e., the equilibrium distribution of $U$ ) and therefore does not contain information on the time scale on which $U(t)$ fluctuates. ${ }^{15,18}$

Another set of three functions corresponds to the $2 \mathrm{PE}$ response to pulses $a$ and $b\left[K\left(t_{1}, t_{2}+t\right)\right]$, pulses $a$ and $c$ $\left[K\left(t_{1}+t_{2}, t\right)\right]$, and pulses $b$ and $c\left[K\left(t_{2}, t\right)\right]$. These three functions indeed also coincide with $K\left(\tau_{1}, \tau\right)$ when plotted as functions of the appropriate time variables (not shown). Two of the remaining three functions correspond to the 3PE response to pulses $a, b$, and $c$ when $t_{1}>0$, i.e., when pulse $a$ precedes pulse $b\left[L_{-}\left(t_{1}, t_{2}, t\right)\right]$ and when $t_{1}<0$, i.e., when pulse $b$ precedes pulse $a\left[L_{+}\left(t_{1}, t_{2}, t\right)\right]$. The last function, $M\left(t_{1}, t_{2}, t\right)$, corresponds to a fifth-order response to the three pulses. Unlike the linear ORF, the decay of these nonlinear ORFs is strongly influenced by fluctuations in $U(t)$ and therefore conveys information on the time scale of solvation dynamics. ${ }^{15,18}$

Comparing Figs. 1 and 2, it is clear that the signals in Fig. 1 are dominated by the linear ORFs $J\left(t_{1}+t_{2}+t\right), J\left(t_{2}\right.$ 


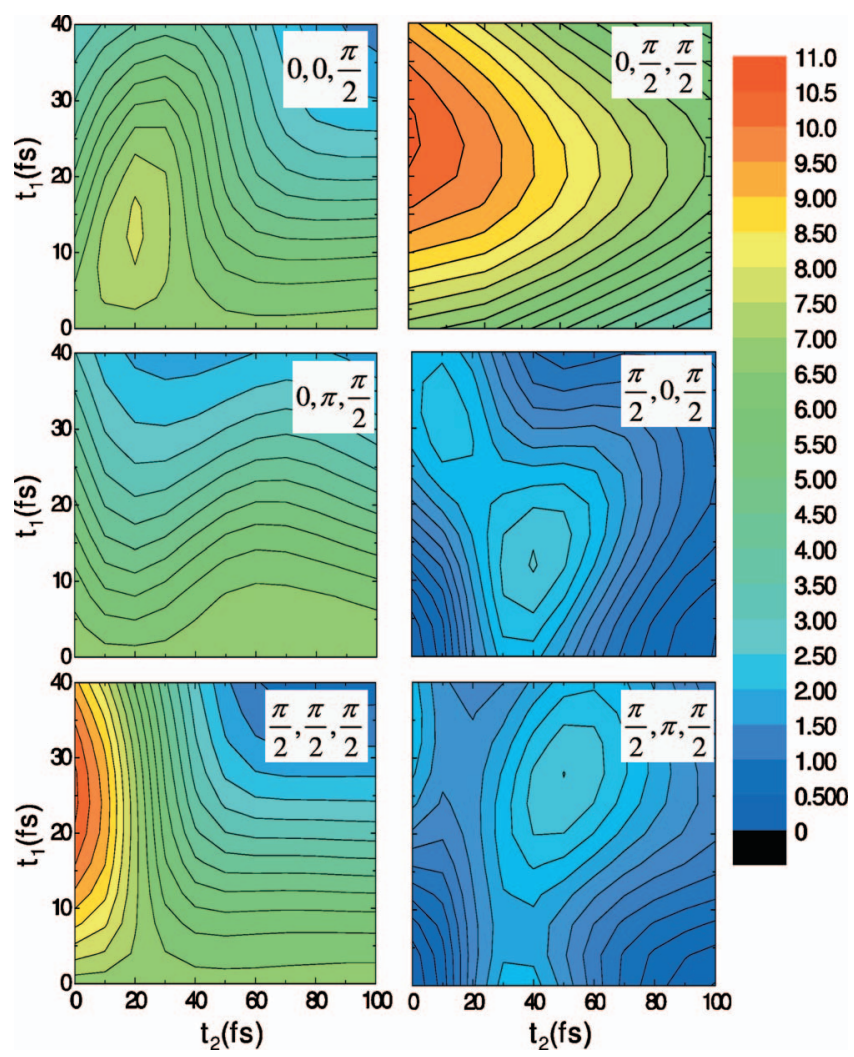

FIG. 3. (Color) The homodyne-detected integrated overall polarizations, $\int_{0}^{\infty} d t\left|\widetilde{\sigma}_{g e}\left(t_{1}, t_{2}, t\right)\right|^{2}$, following a sequence of three impulsive pulses with the indicated six different phase combinations $\left(\phi_{a}, \phi_{b}, \phi_{c}\right)$ [cf. Eq. (28)], under strong-field conditions $\left(\left|\chi_{a}\right| \tau_{a}=\left|\chi_{b}\right| \tau_{b}=\left|\chi_{c}\right| \tau_{c}=\pi / 2\right)$.

$+t$ ), and $J(t)$ (cf. the upper three panels in Fig. 2). This is to be expected under weak-field conditions, where the contribution of higher order ORFs is much smaller in comparison to that of the linear ORFs [cf. Eq. (27)]. Accordingly, the amplitude of the linear ORF that results from Eq. (B1) is much larger than that of the higher order ORFs (prior to dividing by the corresponding field-dependent coefficients). The procedure outlined in Eqs. (26) and (B1) was nevertheless able to extract these much smaller contributions in an accurate manner, as can be seen from Fig. 2. This is surprising at first sight, since the nonlinear signals in Fig. 2 correspond to very small numbers extracted by taking the difference between very large numbers and could be expected to be very sensitive to noise. However, this is not the case due to the phase sensitivity of the signals, which makes this procedure practically noise-free (similarly to experiment).

We next consider another set of results obtained in the strong impulsive pulse regime, under strong-field conditions $\left(\left|\chi_{a}\right| \tau_{a}=\left|\chi_{b}\right| \tau_{b}=\left|\chi_{c}\right| \tau_{c}=\pi / 2\right)$. Figure 3 shows the integrated homodyne-detected overall polarizations following a sequence of three impulsive pulses with the six phase combinations indicated in Eq. (28). Substituting these overall polarizations into Eq. (B2) yields the following six functions: $J\left(t_{1}+t_{2}+t\right), K\left(t_{1}, t_{2}+t\right), K\left(t_{1}+t_{2}, t\right), L_{+}\left(t_{1}, t_{2}, t\right), L_{-}\left(t_{1}, t_{2}, t\right)$, and $M\left(t_{1}, t_{2}, t\right)$. The modulus squares of these functions integrated over $t$ are presented in Fig. 4 and clearly coincide with the same ORFs that were calculated under weak-field conditions (cf. Fig. 2). Comparison of Figs. 3 and 4 also shows
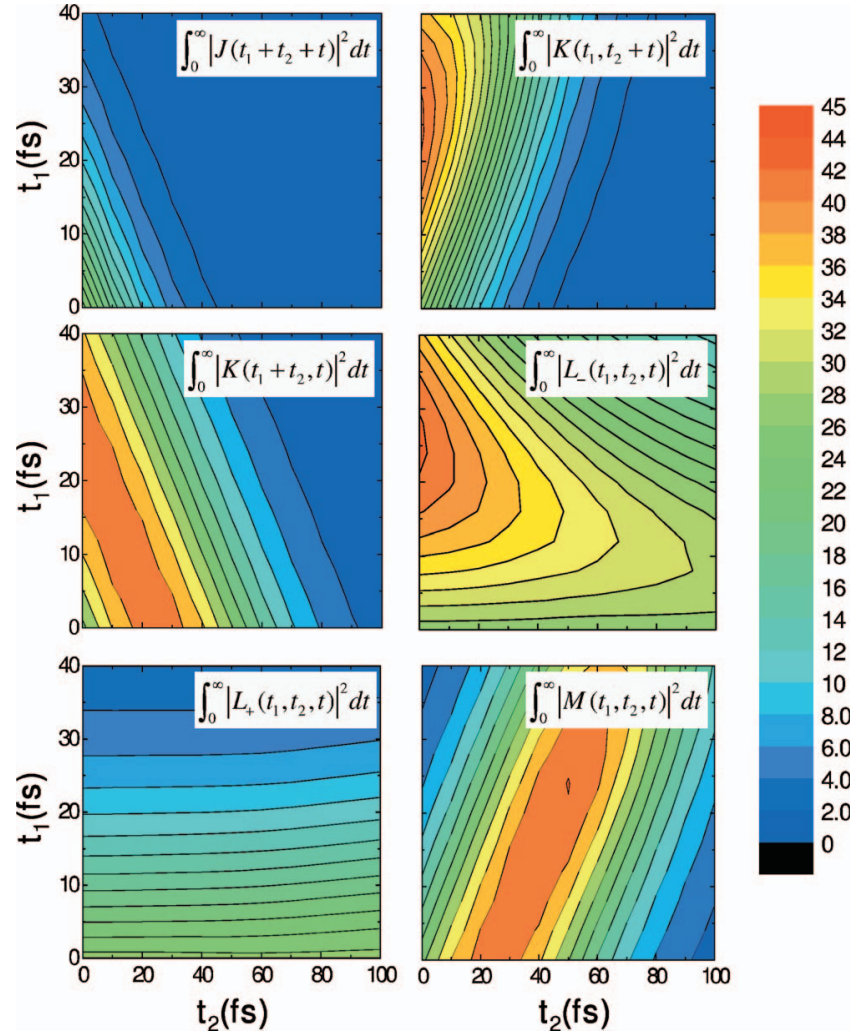

FIG. 4. (Color) The six homodyne-detected integrated ORFs as obtained via Eq. (B2), using as input the six overall polarizations from Fig. 3.

that the signals in Fig. 3 are no longer dominated by the linear ORF, which is to be expected under strong-field conditions.

Another set of results that was generated in the strong impulsive regime and under strong-field conditions $\left(\left|\chi_{a}\right| \tau_{a}\right.$ $\left.=\left|\chi_{b}\right| \tau_{b}=\left|\chi_{c}\right| \tau_{c}=\pi / 2\right)$ is shown in Figs. 5 and 6. Figure 5 shows the integrated homodyne-detected overall polariza-
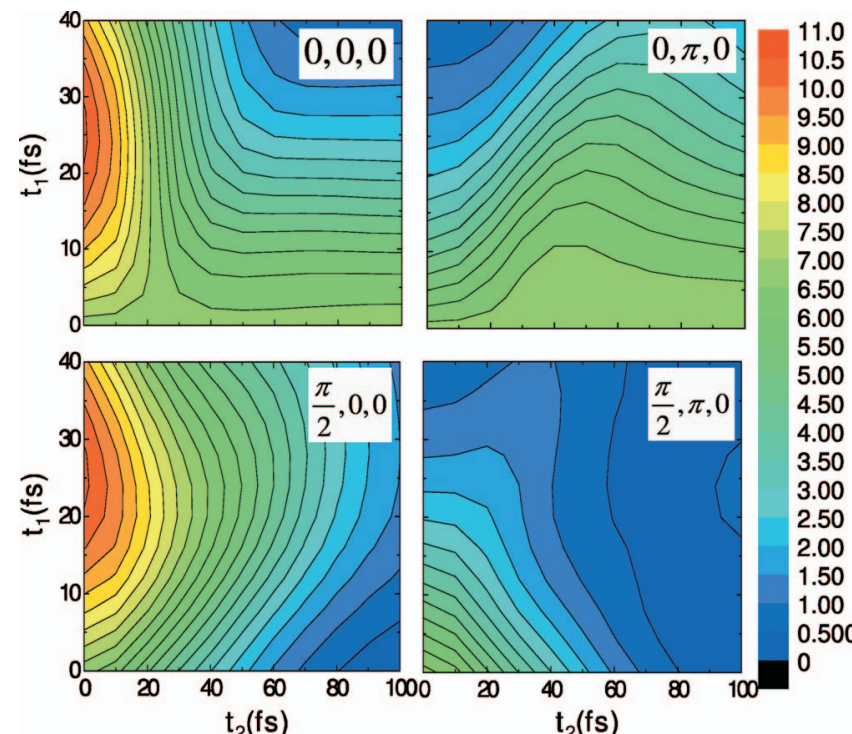

FIG. 5. (Color) The homodyne-detected integrated overall polarizations, $\int_{0}^{\infty} d t\left|\widetilde{\sigma}_{g e}\left(t_{1}, t_{2}, t\right)\right|^{2}$, following a sequence of three impulsive pulses with the indicated four different phase combinations $\left(\phi_{a}, \phi_{b}, \phi_{c}\right)$ [cf. Eq. (29)], under strong-field conditions $\left(\left|\chi_{a}\right| \tau_{a}=\left|\chi_{b}\right| \tau_{b}=\left|\chi_{c}\right| \tau_{c}=\pi / 2\right)$. 


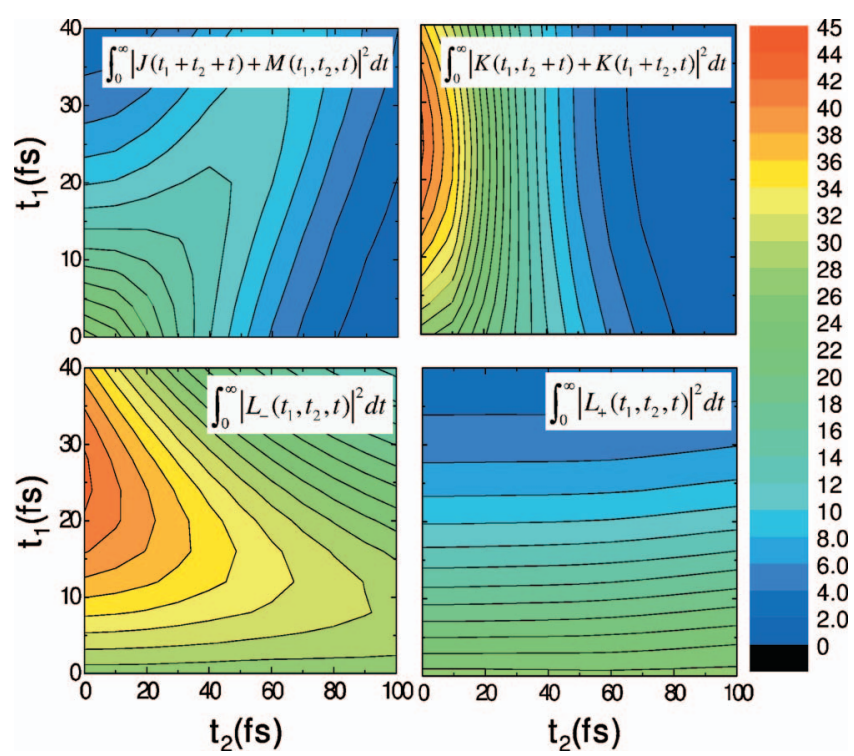

FIG. 6. (Color) The four homodyne-detected integrated ORFs as obtained via Eq. (B3), using as input the four overall polarizations from Fig. 5.

tions following a sequence of three impulsive pulses with the four phase combinations indicated in Eq. (29). Substituting these overall polarizations into Eq. (B3) yields the two 3PE ORFs, $L_{+}\left(t_{1}, t_{2}, t\right)$, and $L_{-}\left(t_{1}, t_{2}, t\right)$, as well as mixtures of $J\left(t_{1}+t_{2}+t\right)$ with $M\left(t_{1}, t_{2}, t\right)$ and of $K\left(t_{1}, t_{2}+t\right)$ with $K\left(t_{1}\right.$ $\left.+t_{2}, t\right)$. The modulus squares of these functions integrated over $t$ are presented in Fig. 6 . The ones for $L_{+}\left(t_{1}, t_{2}, t\right)$ and $L_{-}\left(t_{1}, t_{2}, t\right)$ clearly coincide with these in Figs. 2 and 4.

We now turn to the weak impulsive regime [cf. Eq. (15)]. The difference between the strong and weak impulsive limits has to do with whether one does or does not ignore $U$ during the pulse. The typical amplitude of the fluctuation in $U / \hbar$ around its average value can be estimated in terms of its standard deviation, which is $\sim 0.04 \mathrm{fs}^{-1}$. For the model employed here, the width of an impulsive pulse coincides, by definition, with the MD simulation time step, i.e., $\tau=1.0 \mathrm{fs}$. Thus, for an impulsive pulse, $|U| \tau / \hbar \sim 0.025 \pi / 2$. Thus, under weak-field conditions $(|\chi| \tau \sim 0.01 \pi / 2)$, one cannot assume that $\hbar|\chi| \gg|U|$, which implies that, strictly speaking, the strong impulsive limit is not valid. Nevertheless, we have found that the nine ORFs calculated in the weak impulsive regime and under weak-field conditions $\left(\left|\chi_{a}\right| \tau_{a}=\left|\chi_{b}\right| \tau_{b}\right.$ $\left.=\left|\chi_{c}\right| \tau_{c}=0.01 \pi / 2\right)$ were practically indistinguishable from those obtained in the strong impulsive limit (cf. Figs. 1 and 2). This implies that, at least for the model considered here, accounting for the effect of $U$ during an impulsive pulse does not change the predicted optical response of the system in a noticeable way. The same result was seen to hold for even weaker fields $\left(\left|\chi_{a}\right| \tau_{a}=\left|\chi_{b}\right| \tau_{b}=\left|\chi_{c}\right| \tau_{c}=0.001 \pi / 2\right)$, as well as under strong-field conditions (not shown).

\section{The nonimpulsive regime}

Finally, we consider an example in the nonimpulsive regime. For the sake of convenience, we still assume that the pulses are square but increase their width from 1.0 to $10 \mathrm{fs}$ while lowering their amplitude by a factor of 10 so that the pulse area remains the same as in the impulsive limit. We use the same procedure for extracting the various ORFs as in the impulsive regime, in parallel with the common practice of analyzing experimental signals obtained using nonimpulsive pulses within a theoretical framework that assumes impulsive pulses. We also note that $t_{1}$ was defined as the time interval between the end of pulse $a$ and the end of pulse $b, t_{2}$ as the time interval between the end of pulse $b$ and the end of pulse $c$, and $t$ as the time interval between the end of pulse $c$, and detection.

The ORFs calculated in the nonimpulsive regime under weak-field conditions $\left(\left|\chi_{a}\right| \tau_{a}=\left|\chi_{b}\right| \tau_{b}=\left|\chi_{c}\right| \tau_{c}=0.01 \pi / 2\right)$ and strong-field conditions $\left(\left|\chi_{a}\right| \tau_{a}=\left|\chi_{b}\right| \tau_{b}=\left|\chi_{c}\right| \tau_{c}=\pi / 2\right) \quad$ are shown in Figs. 7 and 8, respectively. As can be expected, comparison with the corresponding results obtained in the impulsive regime (cf. Figs. 2 and 4, respectively) reveals many quantitative and qualitative differences, particularly at $t_{1}, t_{2} \leqslant 10$ fs (i.e., when there is significant overlap between pulses). Furthermore, unlike in the impulsive regime, the ORFs extracted under strong-field conditions are noticeably different from those extracted under weak-field conditions. However, many of the gross features remain similar in both cases. For example, a comparison of the 3PE ORF, $L_{-}\left(t_{1}, t_{2}, t\right)$, in the impulsive and nonimpulsive regimes reveals that the decay of the signal as a function of $t_{2}$ occurs on similar time scales, while the maximum as a function of $t_{1}$ is shifted to a value of $t_{1}$ which is about $10 \mathrm{fs}$ longer than in the nonimpulsive case.

\section{CONCLUDING REMARKS}

In this paper, we presented and demonstrated a nonperturbative procedure for calculating nonlinear optical signals in liquid solution. It should be noted that although the presentation was made in the context of electronic spectroscopy, the same procedure will apply equally well in the case of vibrational spectroscopy. It should also be emphasized that the procedure can be easily extended to cases involving multiple electronic and/or vibrational transitions. Being nonperturbative, the proposed procedure is clearly advantageous in the strong-field regime. However, it also appears to provide a convenient alternative to the perturbative treatment in the weak-field regime.

The nonperturbative approach is particularly advantageous in the nonimpulsive regime. Although we have only considered the effect of using square pulses of finite width, one could employ the very same scheme in order to understand the effect of using nonimpulsive laser pulses of more complex shapes. For example, a nonperturbative approach of the type described here would be very useful in exploring the prospects of using shaped laser pulses to control molecular dynamics in liquid solution. ${ }^{41-60}$

In calculating the overall polarization and only then resolving it into its directional components, the nonperturbative approach follows a procedure which is similar to the 

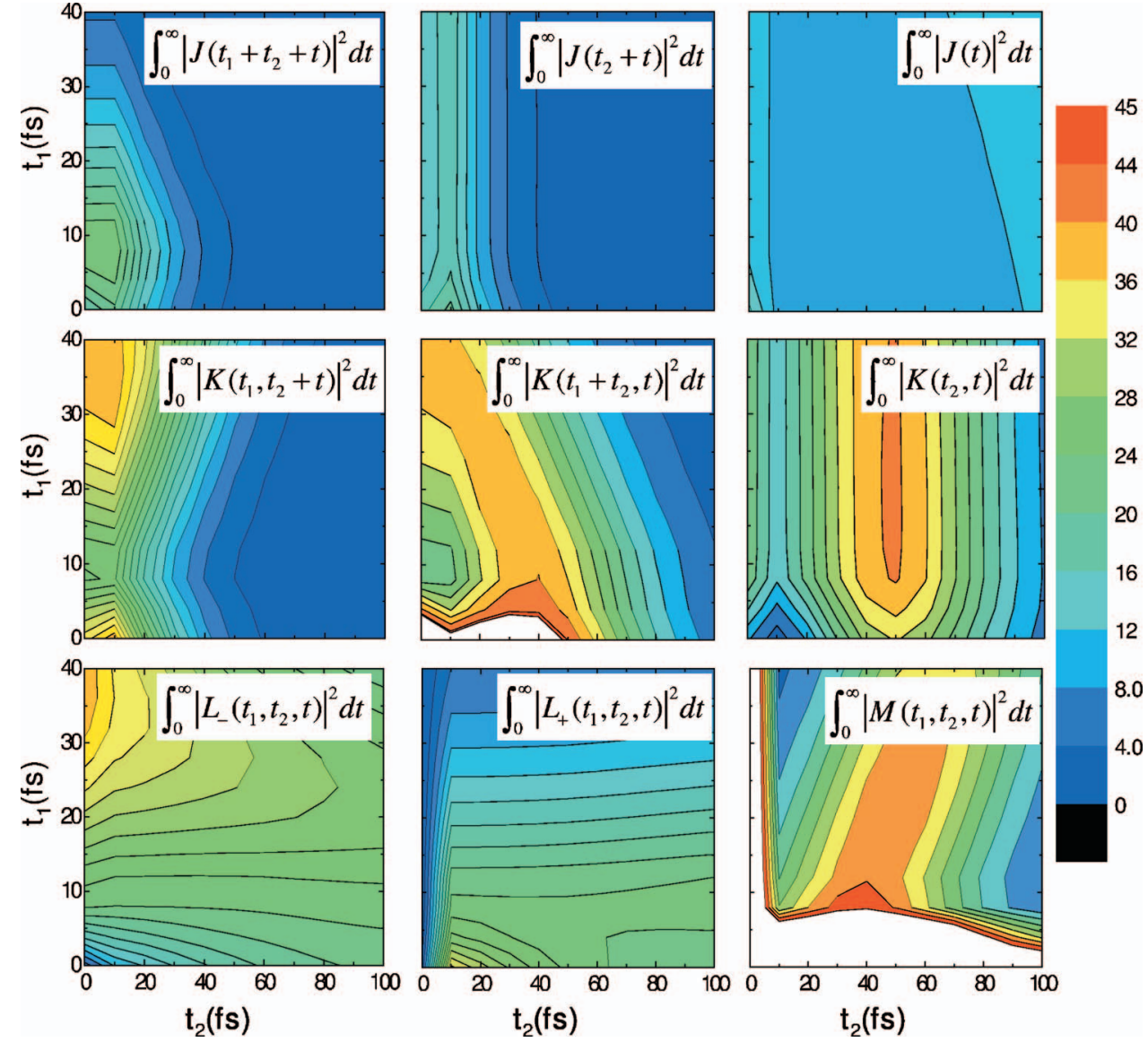

experimental one. The nonperturbative approach also highlights the fact that, in the impulsive regime, the overall polarization is given by a linear combination of the same ORFs, regardless of the field strength. As a result, choosing between the strong-field and the weak-field regimes becomes a matter of convenience. In fact, the strong-field route to the ORFs turns out to be somewhat more convenient in practice.

In resolving the overall polarization into its directional components in the nonimpulsive case, we assumed that it consists of the same nine directional components, as in the impulsive case. Our goal in pursuing this calculation was to critically examine the quality of the results obtained by analyzing experimental signals obtained using nonimpulsive pulses within a theoretical framework that assumes impulsive pulses. It should be emphasized, however, that the actual overall polarization measured in a nonimpulsive three-pulse experiment involves additional components. Thus, in general, the overall polarization will include components associated with phase factors of the following form: $\exp \left[i\left(n_{a} \phi_{a}\right.\right.$ $\left.\left.+n_{b} \phi_{b}+n_{c} \phi_{c}\right)\right]$, with $n_{a}, n_{b}, n_{c}=0, \pm 1, \pm 2, \pm 3, \ldots$ (although the corresponding components for some phase combinations may vanish). ${ }^{29}$ Within the weak-field regime, higher values of $\left(n_{a}, n_{b}, n_{c}\right)$ are associated with higher order with respect to the field, which would simplify the interpretation of nonlinear signals.

The scheme considered here was based on several simplifying assumptions such as the rotating wave approximation, the same leading frequency for all pulses, and resonance. However, none of these assumptions is essential, and they can all be avoided in principle (at the expense of addi-

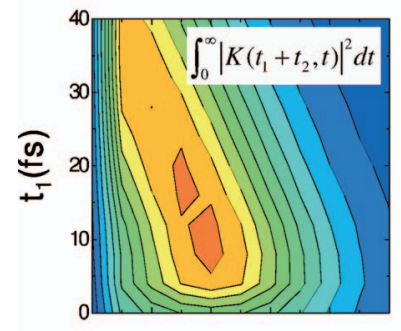
$\left(\left|\chi_{a}\right| \tau_{a}=\left|\chi_{b}\right| \tau_{b}=\left|\chi_{c}\right| \tau_{c}=\pi / 2\right)$.
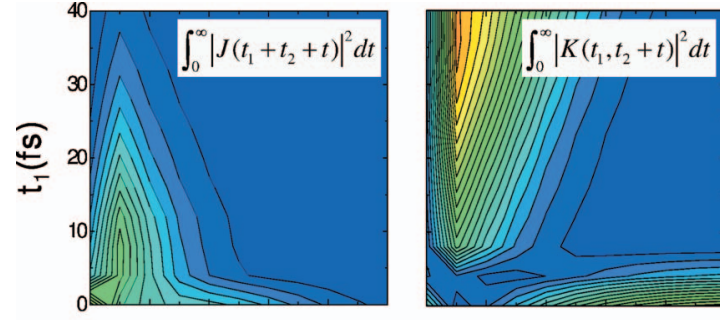

FIG. 7. (Color) The nine homodynedetected integrated ORFs calculated via Eq. (B1), using as input the overall polarizations following a sequence of three nonimpulsive $10 \mathrm{fs}$ wide square pulses with the nine different phase combinations $\left(\phi_{a}, \phi_{b}, \phi_{c}\right)$ specified in Eq. (28), under weak-field conditions $\left(\left|\chi_{a}\right| \tau_{a}=\left|\chi_{b}\right| \tau_{b}=\left|\chi_{c}\right| \tau_{c}=0.01 \pi / 2\right)$.

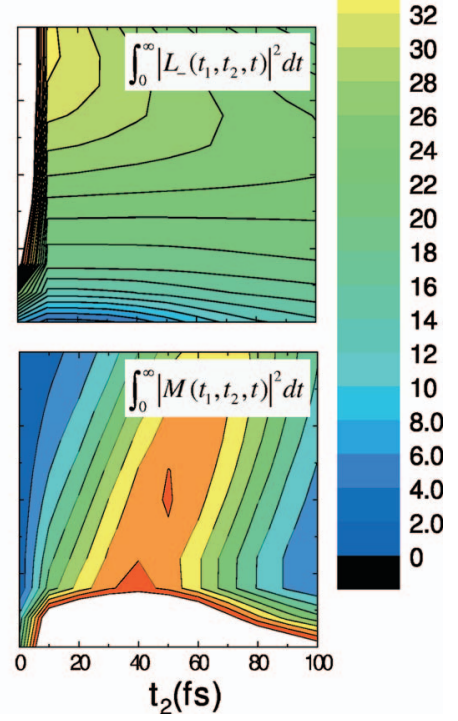

FIG. 8. (Color) The six homodyne-detected integrated ORFs calculated via Eq. (B2), using as input the overall polarizations following a sequence of three nonimpulsive $10 \mathrm{fs}$ wide square pulses with the six different phase combinations $\left(\phi_{a}, \phi_{b}, \phi_{c}\right)$ specified in Eq. (28), under strong-field conditions 
tional computational cost). A more significant limitation of the proposed scheme has to do with the fact that the mixed quantum-classical treatment that we employed, which was dictated by the specific choice of interaction picture implied by Eq. (7), is not unique. As is well known, adopting other quantum-mechanically equivalent interaction pictures and following a similar procedure would lead to different mixed quantum-classical treatments (cf. Appendix A). ${ }^{10-17}$ At the same time, a previous study has shown that the nonlinear signals are not very sensitive to the type of approximation employed for describing the dynamics in the case of a nonpolar solution. ${ }^{23}$ However, a more detailed study of this issue in nonpolar as well as polar solutions would be highly desirable and will be the subject of future work.

\section{ACKNOWLEDGMENTS}

This project was supported by the National Science Foundation, through Grant No. CHE-306695 and The Petroleum Research Fund.

\section{APPENDIX A: ALTERNATIVE MIXED QUANTUM-CLASSICAL SCHEMES}

The mixed quantum-classical treatment that we employed is dictated by the specific partitioning of the Hamiltonian in Eq. (2), which in turn dictates the choice of interaction picture implied by Eq. (7). Although this is a convenient choice (see below), it is not unique. To see why, consider the following more general partitioning of the Hamiltonian in Eq. (2):

$$
\hat{H}_{0}=\hbar \omega_{e g}|e\rangle\langle e|+\hat{H}_{b}+\hat{U}[\alpha|e\rangle\langle e|-(1-\alpha)| g\rangle\langle g|]
$$

where $\hat{H}_{b}=\alpha \hat{H}_{g}+(1-\alpha) \hat{H}_{e}$ and $0 \leqslant \alpha \leqslant 1$. It should be noted that the partitioning in Eq. (2) is reproduced for $\alpha=1$. We next define the transformation to the rotating frame and interaction picture as follows:

$$
\widetilde{\rho}(t)=\exp \left[i\left(\hat{H}_{b}+\hbar \omega|e\rangle\langle e|\right) t / \hbar\right] \hat{\rho}(t) \exp \left[-i\left(\hat{H}_{b}+\hbar \omega|e\rangle\langle e|\right) t / \hbar\right]
$$

It can be easily shown that the equation of motion for $\widetilde{\rho}(t)$ has the form of Eq. (8), with

$$
\tilde{H}(t)=\hbar \Delta|e\rangle\langle e|+\hat{U}_{b}(t)[\alpha|e\rangle\langle e|-(1-\alpha)| g\rangle\langle g|]-\frac{\hbar}{2} \sum_{j}\left(\chi_{j}(t)|g\rangle\left\langle e\left|+\chi_{j}^{*}(t)\right| e\right\rangle\langle g|\right)
$$

Here, $\hat{U}_{b}(t)=e^{i \hat{H}_{b} t / \hbar} \hat{U} e^{-i \hat{H}_{b} t / \hbar}$.

Substituting $\hat{U}_{b}(t)$ by its classical limit, $U_{b}(t)$, it can be easily shown that the coupled equations of motion for the four elements of the density matrix $\widetilde{\sigma}(t)$ are still given by Eqs. (12) and (13). However, $U_{b}(t)$ is different from $U(t)$ and corresponds to the energy gap between the ground and excited electronic states as obtained from a classical nonequilibrium MD simulation on the averaged potential surface $\alpha V_{g}+(1-\alpha) V_{e}$.

The fact that $U_{b}(t)$ is explicitly dependent on $\alpha$ implies that equivalent forms of the quantum Hamiltonian give rise to different mixed quantum schemes that differ with respect to the potential surface that dictates the dynamics of $U_{b}(t) .{ }^{10-17}$ The choice $\alpha=1$, for which $U_{b}(t)=U(t)$, is therefore somewhat $a d$ hoc. However, the choice of $\alpha=1$ is also convenient since unlike any other choice, it allows us to extract $U(t)$ from equilibrium MD simulations (on the ground state potential surface). It should also be noted that a previous study has shown that the nonlinear signals are relatively insensitive to the choice of $\alpha$ in the case of the nonpolar solution model considered here. ${ }^{23}$

\section{APPENDIX B: INVERSE TRANSFORMATIONS}

The inverse of Eq. (26) is given by 


$$
\begin{aligned}
& \left(\begin{array}{c}
a_{1} J\left(t_{1}+t_{2}+t\right) \\
a_{2} J\left(t_{2}+t\right) \\
a_{3} J(t) \\
b_{1} K\left(t_{1}, t_{2}+t\right) \\
b_{2} K\left(t_{1}+t_{2}, t\right) \\
b_{3} K\left(t_{2}, t\right) \\
c_{1} L_{+}\left(t_{1}, t_{2}, t\right) \\
c_{2} L_{-}\left(t_{1}, t_{2}, t\right) \\
d M\left(t_{1}, t_{2}, t\right)
\end{array}\right)=\frac{1}{8}\left(\begin{array}{ccccccccc}
i & 1+i & 1 & 1-i & -2 i & -1-i & -1 & -1+i & i \\
1+i & -2 i & -1+i & 0 & 0 & 0 & 1+i & -2 i & -1+i \\
-2 i & 0 & -2 i & 0 & 0 & 0 & -2 i & 0 & -2 i \\
1 & -1+i & -i & -1+i & -2 i & 1+i & -i & 1+i & -1 \\
i & -1+i & -1 & -1-i & -2 i & 1-i & 1 & 1+i & i \\
-1+i & -2 i & 1+i & 0 & 0 & 0 & -1+i & -2 i & 1+i \\
1-i & 0 & -1+i & -2 & 0 & 2 & 1+i & 0 & -1-i \\
-1-i & 0 & 1+i & 2 & 0 & -2 & -1+i & 0 & 1-i \\
-1 & 1+i & -i & 1+i & -2 i & -1+i & -i & -1+i & 1
\end{array}\right) \\
& \times\left(\begin{array}{l}
\widetilde{\sigma}_{g e}^{00(\pi / 2)}\left(t_{1}, t_{2}, t\right) \\
\widetilde{\sigma}_{g e}^{0(\pi / 2)(\pi / 2)}\left(t_{1}, t_{2}, t\right) \\
\widetilde{\sigma}_{g e}^{0 \pi(\pi / 2)}\left(t_{1}, t_{2}, t\right) \\
\widetilde{\sigma}_{g e}^{(I / 2) 0(\pi / 2)}\left(t_{1}, t_{2}, t\right) \\
\widetilde{\sigma}_{g e}^{(I / 2)(\pi / 2)(\pi / 2)}\left(t_{1}, t_{2}, t\right) \\
\widetilde{\sigma}_{g e}^{(\pi / 2) \pi(\pi / 2)}\left(t_{1}, t_{2}, t\right) \\
\widetilde{\sigma}_{g e}^{\pi 0(\pi / 2)}\left(t_{1}, t_{2}, t\right) \\
\widetilde{\sigma}_{g e}^{\pi(\pi / 2)(\pi / 2)}\left(t_{1}, t_{2}, t\right) \\
\widetilde{\sigma}_{g e}^{\pi \pi(\pi / 2)}\left(t_{1}, t_{2}, t\right)
\end{array}\right)
\end{aligned}
$$

The inverse of Eq. (28) is given by

$$
\left(\begin{array}{c}
J\left(t_{1}+t_{2}+t\right) \\
K\left(t_{1}, t_{2}+t\right) \\
K\left(t_{1}+t_{2}, t\right) \\
L_{+}\left(t_{1}, t_{2}, t\right) \\
L_{-}\left(t_{1}, t_{2}, t\right) \\
M\left(t_{1}, t_{2}, t\right)
\end{array}\right)=\left(\begin{array}{cccccc}
-1+i & 2 i & 1+i & 1+i & 2 & 1-i \\
1-i & 2 i & -1-i & 1+i & -2 & 1-i \\
1+i & 2 i & -1+i & -1+i & -2 & -1-i \\
-1 & 0 & 1 & i & 0 & -i \\
-1 & 0 & 1 & -i & 0 & i \\
-1-i & 2 i & 1-i & -1+i & 2 & -1-i
\end{array}\right)\left(\begin{array}{l}
\widetilde{\sigma}_{g e}^{00(\pi / 2)}\left(t_{1}, t_{2}, t\right) \\
\widetilde{\sigma}_{g e}^{0(\pi / 2)(\pi / 2)}\left(t_{1}, t_{2}, t\right) \\
\widetilde{\sigma}_{g e}^{0 \pi(\pi / 2)}\left(t_{1}, t_{2}, t\right) \\
\widetilde{\sigma}_{g e}^{(\pi / 2) 0(\pi / 2)}\left(t_{1}, t_{2}, t\right) \\
\widetilde{\sigma}_{g e}^{(\pi / 2)(\pi / 2)(\pi / 2)}\left(t_{1}, t_{2}, t\right) \\
\widetilde{\sigma}_{g e}^{(\pi / 2) \pi(\pi / 2)}\left(t_{1}, t_{2}, t\right)
\end{array}\right) .
$$

The inverse of Eq. (29) is given by

$$
\left(\begin{array}{c}
J\left(t_{1}+t_{2}+t\right)+M\left(t_{1}, t_{2}, t\right) \\
K\left(t_{1}, t_{2}+t\right)+K\left(t_{1}+t_{2}, t\right) \\
L_{+}\left(t_{1}, t_{2}, t\right) \\
L_{-}\left(t_{1}, t_{2}, t\right)
\end{array}\right)=\left(\begin{array}{cccc}
2 i & 2 i & 2 & 2 \\
-2 i & -2 i & 2 & 2 \\
-i & i & -1 & 1 \\
-i & i & 1 & -1
\end{array}\right)\left(\begin{array}{l}
\widetilde{\sigma}_{g e}^{000}\left(t_{1}, t_{2}, t\right) \\
\widetilde{\sigma}_{g e}^{0 \pi 0}\left(t_{1}, t_{2}, t\right) \\
\widetilde{\sigma}_{g e}^{(\pi / 2) 00}\left(t_{1}, t_{2}, t\right) \\
\widetilde{\sigma}_{g e}^{(\pi / 2) \pi 0}\left(t_{1}, t_{2}, t\right)
\end{array}\right) .
$$

${ }^{1}$ S. Mukamel, Principles of Nonlinear Optical Spectroscopy (Oxford, New York, 1995).

${ }^{2}$ G. R. Fleming and M. Cho, Annu. Rev. Phys. Chem. 47, 109 (1996).

${ }^{3}$ W. P. de Boeij, M. S. Pshenichnikov, and D. A. Wiersma, Annu. Rev. Phys. Chem. 49, 99 (1998).

${ }^{4}$ A. B. Myers, Annu. Rev. Phys. Chem. 49, 267 (1998).

${ }^{5}$ R. M. Stratt and M. Maroncelli, J. Phys. Chem. 100, 12981 (1996).

${ }^{6}$ P. Vöhringer, D. C. Arnett, T.-S. Yang, and N. F. Scherer, Chem. Phys. Lett. 237, 387 (1995).

${ }^{7}$ W. Domcke and G. Stock, Adv. Chem. Phys. 100, 1 (1997).

${ }^{8}$ D. M. Jonas, Annu. Rev. Phys. Chem. 54, 425 (2003).

${ }^{9}$ M. Cho, S. J. Rosenthal, N. F. Scherer, L. D. Ziegler, and G. R. Fleming, J. Chem. Phys. 96, 5618 (1992).

${ }^{10}$ S. Mukamel, J. Chem. Phys. 77, 173 (1982).

${ }^{11}$ N. E. Shemetulskis and R. F. Loring, J. Chem. Phys. 97, 1217 (1992).

${ }^{12}$ B. D. Bursulaya and H. J. Kim, J. Phys. Chem. 100, 16451 (1996).

${ }^{13}$ L. E. Fried, N. B. Bernstein, and S. Mukamel, Phys. Rev. Lett. 68, 1842 (1992).

${ }^{14}$ J. G. Saven and J. L. Skinner, J. Chem. Phys. 99, 4391 (1993).
${ }^{15}$ M. D. Stephens, J. G. Saven, and J. L. Skinner, J. Chem. Phys. 106, 2129 (1997).

${ }^{16}$ S. A. Egorov, E. Rabani, and B. J. Berne, J. Chem. Phys. 108, 1407 (1998).

${ }^{17}$ K. F. Everitt, E. Geva, and J. L. Skinner, J. Chem. Phys. 114, 1326 (2001).

${ }^{18}$ K. F. Everitt and J. L. Skinner, Chem. Phys. 266, 197 (2001).

${ }^{19}$ J. L. Skinner, J. Chem. Phys. 77, 3398 (1982).

${ }^{20}$ A. M. Walsh and R. F. Loring, Chem. Phys. Lett. 186, 77 (1991).

${ }^{21}$ O. Kühn and N. Makri, J. Phys. Chem. A 103, 9487 (1999).

${ }^{22}$ M. Ovchinnikov, V. A. Apkarian, and G. A. Voth, J. Chem. Phys. 184, 7130 (2001).

${ }^{23}$ Q. Shi and E. Geva, J. Chem. Phys. 122, 064506 (2005).

${ }^{24}$ A. D. Hammerich, R. Kosloff, and M. A. Ratner, J. Chem. Phys. 97, 6410 (1992).

${ }^{25}$ U. Banin, A. Bartana, S. Ruhman, and R. Kosloff, J. Chem. Phys. 101, 8461 (1994).

${ }^{26}$ G. Ashkenazi, U. Banin, A. Bartana, R. Kosloff, and S. Ruhman, Adv. Chem. Phys. 100, 229 (1997). 
${ }^{27}$ M. Sugawara1 and Y. Fujimura, Chem. Phys. 175, 323 (1993).

${ }^{28}$ G. Stock and W. Domcke, Phys. Rev. A 45, 3032 (1992).

${ }^{29}$ L. Seidner, G. Stock, and W. Domcke, J. Chem. Phys. 103, 3998 (1995).

${ }^{30}$ B. Wolfseder, L. Seidner, G. Stock, and W. Domcke, Chem. Phys. 217, 275 (1997).

${ }^{31}$ M. F. Gelin, A. V. Pisliakov, D. Egorova, and W. Domcke, J. Chem. Phys. 118, 5287 (2003)

${ }^{32}$ M. F. Gelin, D. Egorova, and W. Domcke, J. Chem. Phys. 23, 164112 (2005).

${ }^{33}$ J. Faeder, I. Pinkas, G. Knopp, Y. Prior, and D. J. Tannor, J. Chem. Phys. 115, 8440 (2001).

${ }^{34}$ C. P. Koch, T. Klüner, and R. Kosloff, J. Chem. Phys. 116, 7983 (2002)

${ }^{35}$ D. Gelman, G. Katz, R. Kosloff, and M. A. Ratner, J. Chem. Phys. 123, $134112(2005)$

${ }^{36}$ T. I. C. Jansen, J. G. Snijders, and K. Duppen, J. Chem. Phys. 113, 307 (2000).

${ }^{37}$ T. I. C. Jansen, J. G. Snijders, and K. Duppen, J. Chem. Phys. 114, 10910 (2001).

${ }^{38}$ T. Mančal, A. V. Pisliakov, and G. R. Fleming, J. Chem. Phys. 124, 234504 (2006).

${ }^{39}$ A. V. Pisliakov, T. Mančal, and G. R. Fleming, J. Chem. Phys. 124, 234505 (2006).

${ }^{40}$ M. P. Allen and D. J. Tildesley, Computer Simulation of Liquids (Clarendon, Oxford, 1987).

${ }^{41}$ F. Grossmann, T. Dittrich, P. Jung, and P. Hänggi, Phys. Rev. Lett. 67, 516 (1991).

${ }^{42}$ R. Bavli and H. Metiu, Phys. Rev. Lett. 69, 1986 (1992).
${ }^{43}$ D. G. Evans, R. D. Coalson, H. J. Kim, and Y. Dakhnovskii, Phys. Rev. Lett. 75, 3649 (1995).

${ }^{44}$ A. Bartana, R. Kosloff, and D. J. Tannor, J. Chem. Phys. 99, 196 (1993).

${ }^{45}$ A. Bartana, R. Kosloff, and D. J. Tannor, J. Chem. Phys. 106, 1435 (1997).

${ }^{46}$ D. E. Makarov and N. Makri, Phys. Rev. B 52, R2257 (1995).

${ }^{47}$ N. Makri, J. Chem. Phys. 106, 2286 (1997).

${ }^{48}$ N. Makri and L. Wei, Phys. Rev. E 55, 2475 (1997).

${ }^{49}$ R. I. Cukier, C. Denk, and M. Morillo, Chem. Phys. 217, 179 (1997).

${ }^{50}$ M. Grifoni and P. Hänggi, Phys. Rep. 304, 229 (1998).

${ }^{51}$ O. Kühn, Eur. Phys. J. D 6, 49 (1999).

${ }^{52}$ H. Naundorf, K. Sundermann, and O. Kühn, Chem. Phys. 240, 163 (1999).

${ }^{53}$ O. Kuhn, Y. Zhao, F. Shuang, and Y. Yan, J. Chem. Phys. 112, 6104 (2000).

${ }^{54}$ N. Dŏslić, O. Kühn, J. Manz, and K. Sundermann, J. Phys. Chem. A 102, 9645 (1998)

${ }^{55}$ N. Dŏslić, K. Sunderman, L. González, O. Mó, J. Giraud-Girard, and O. Kühn, Phys. Chem. Chem. Phys. 1, 1249 (1999).

${ }^{56}$ T. C. Weinacht, J. L. White, and P. H. Bucksbaum, J. Phys. Chem. A 103, 10166 (1999).

${ }^{57}$ E. Geva, J. Chem. Phys. 116, 1629 (2001).

${ }^{58}$ M. Demirplak and S. A. Rice, J. Chem. Phys. 116, 8028 (2002).

${ }^{59}$ Y. Ohtsuki, K. Nakagami, W. Zhu, and H. Rabitz, Chem. Phys. 287, 197 (2003).

${ }^{60}$ Q. Shi and E. Geva, J. Chem. Phys. 119, 11773 (2003). 